\author{
典型芳香烃大气氧化机理研究进展 \\ 宋梦迪刘莹李歆* 陆思华 \\ (北京大学环境科学与工程学院 环境模拟与污染控制国家重点联合实验室 \\ 教育部区域污染控制国际合作联合实验室＼cjkstart北京 100871)
}

\begin{abstract}
摘要 芳香烃作为城市大气臭氧 $\left(\mathrm{O}_{3}\right)$ 和二次有机气溶胶 $(\mathrm{SOA})$ 的重要前体物, 由于其对大气二次污染、气候变化及人体 健康具有重要影响, 因此芳香烃氧化机理研究成为当前大气环境化学领域最具挑战的热点研究之一. 本文综述了芳香 烃氧化机理的研究成果, 详细讨论了它们与 $\mathrm{OH}$ 自由基在高低氮氧化物条件下的各反应通道和影响因素, 重点关注近 年来芳香烃氧化反应研究中的新发现和新理论. 芳香烃的大气氧化反应起始由 $\mathrm{OH}$ 自由基主导, 根据其反应产物主要 分为醛通道、酚通道、双环 $\mathrm{RO}_{2}$ 通道和环氧化物通道. 随着形成多差基化合物、烷氧自由基环氧化、双环过氧自由基 分子内氢转移、醛类化合物氢转移、CO-loss 生成低碳产物等新理论的提出, 对芳香烃氧化的理解虽然有所提高, 但反 应过程仍存在碳质量不守恒和自由基不闭合的问题, 导致对后续 $\mathrm{O}_{3}$ 和 SOA 形成机制的认识还十分有限. 理论计算和 实验室模拟是目前芳香烃氧化机理研究的主要手段. 质谱法和光谱法是芳香烃氧化产物最常用的测量技术, 在线质谱 技术尝试从分子水平上捕捉示踪性中间产物的转化, 对揭示芳烃氧化机理具有重要作用. 随着示踪物测量技术的发展, 特别是色谱一质谱联用技术为中间产物的精准测量和芳香烃氧化机制的完善开拓了新方向. 在此基础上, 厘清芳烃氧 化中的碳平衡和自由基收支等关键科学问题、探究实际大气中芳香烃的环境效应有望成为未来该领域的重点研究方向.
\end{abstract} 关键词 芳香烃; 醛途径; 酚途径; 双环 $\mathrm{RO}_{2}$ 途径; 氢转移反应

\title{
Advances on Atmospheric Oxidation Mechanism of Typical Aromatic Hydrocarbons
}

\author{
Mengdi Song Ying Liu Xin Li* Sihua Lu
}

(State Key Joint Laboratory of Environmental Simulation and Pollution Control, International Joint Laboratory for Regional Pollution Control, Ministry of Education (IJRC), College of Environmental Sciences and

Engineering, Peking University, Beijing 100871, China)

\begin{abstract}
Aromatic hydrocarbons are important precursors of ozone and secondary organic aerosols in the urban atmosphere, which have important impact on air pollution, climate change and human health. Thus the research on the oxidation mechanism of aromatic hydrocarbons has become one of the most challenging hotspots topics in atmospheric environmental chemistry. This paper reviews the recent studies of aromatic hydrocarbons oxidation mechanism, discusses the reaction channels and influencing factors during the oxidation process under high/low $\mathrm{NO}_{x}$ conditions, and focuses on new discoveries and new theories in the studies of aromatic hydrocarbon oxidation reactions. In the atmosphere, the initiation of aromatic hydrocarbons is dominated by the reaction with the $\mathrm{OH}$ radicals. According to the different reaction products, the oxidation reaction of aromatic hydrocarbon is mainly divided into aldehyde pathway, phenolic pathway, bicyclic $\mathrm{RO}_{2}$ pathway and epoxide pathway. With the development of new theories such as the formation of polyhydroxy compounds by aldehyde pathway, alkoxy radical epoxidation reaction, intramolecular H-migration reaction of bicyclic peroxy radicals, 1,5-aldehydic $\mathrm{H}$-shift reaction, and the generation of low-carbon products by CO-loss reaction, the understanding of aromatic hydrocarbons oxidation mechanisms has improved. However, due to the carbon missing and radical budgets imbalance problems in the existing oxidation mechanism, our understanding about the subsequent $\mathrm{O}_{3}$ and secondary organic aerosols formation mechanisms are very limited. Theoretical calculation and experiment are the main methods to study the oxidation mechanism of aromatic hydrocarbons. Mass spectrometry and spectroscopy are the dominant measurement techniques for the oxidation intermediates of aromatic hydrocarbons. Online mass spectrometry can capture the tracer intermediates at the molecular level, which plays an important role in revealing the oxidation mechanism of aromatic hydrocarbons. In recent years, tracer measurement technology, especially chromatography-mass spectrometry technology has developed rapidly. This open up a new direction for the accurate measurement of intermediates and the improvement of aromatic hydrocarbon oxidation mechanism. On this basis, improving aromatic hydrocarbons oxidation mechanism, paying attention to the carbon missing and radical budgets imbalance in the oxidation reaction, and exploring the environmental implication of aromatic hydrocarbons oxidation in the real atmospheric conditions are expected to become the important directions of aromatic hydrocarbon oxidation study
\end{abstract}

\footnotetext{
*E-mail: 1i_xin@pku.edu.cn

Received May 20, 2021; published July 30, 2021.

Project supported by the National Natural Science Foundation of China (Nos. 91844301, 91644108).

项目受国家自然科学基金(Nos. 91844301, 91644108)资助.
} 
in the future.

Keywords aromatic hydrocarbon; aldehyde pathway; phenolic pathway; bicyclic $\mathrm{RO}_{2}$ pathway; $\mathrm{H}$-migration

\section{1 引言}

中国现阶段大气污染呈现二次污染频发的特点, 主 要体现在臭氧 $\left(\mathrm{O}_{3}\right)$ 浓度逐年升高, 二次有机气溶胶 $(\mathrm{SOA})$ 组分在 $\mathrm{PM}_{2.5}$ 中占比逐年增高, 日益严峻的二次 污染已成为制约中国环境空气质量持续改善的关键因 素 ${ }^{[1-2]}$. 臭氧和二次有机气溶胶的生成与大气环境中芳 香烃(aromatic hydrocarbons, AHs)的氧化反应是密不可 分的. 芳香烃作为城市大气中挥发性有机化合物的重要 组成部分, 主要包括苯、甲苯、二甲苯和三甲苯等, 约 占总挥发性有机物(VOCs)浓度的 $20 \% \sim 30 \%$ 且呈现逐 年递增状态 ${ }^{[3]}$. 芳香烃通过机动车排放、溶剂使用及工 业排放进入大气环境后 ${ }^{[4-5]}$, 会迅速和 $\mathrm{OH}$ 自由基发生反 应, 伴随着高氧化态低挥发性的中间氧化产物和大量的 $\mathrm{RO}_{x}$ 和 $\mathrm{HO}_{x}$ 自由基的生成(图 1), 与城市地区 $\mathrm{O}_{3}$ 及 PM2.5 的生成密切相关 ${ }^{[6]}$. VOCs 源排放清单结果显示, 在京津冀、长三角和珠三角地区芳香烃对臭氧生成潜势 (OFP) 的贡献占比分别达到 $40.8 \%, 50.4 \%$ 和 $54.6 \%{ }^{[7]}$. 观 测结果显示, 在北京、上海、深圳和成都地区, 芳香烃 对 OFP 的贡献分别约为 $47 \%{ }^{[8]}, 42.7 \%{ }^{[9]}, 65 \% \sim 73 \%{ }^{[10]}$ 和 $24 \%{ }^{[11]}$. 此外, 芳香烃作为对二次有机气溶胶生成潜
势(SOAP)贡献最大的一类挥发性有机物, 主导着城市 的 SOA 形成. 源清单结果显示, 在京津冀、长三角、珠 三角和成渝地区芳香烃对 SOAP 的贡献占比分别达到 $67.5 \%, 71.8 \%, 70.5 \%$ 和 $67.9 \%{ }^{[12]}$. 实验室结果也指出, 苯、甲苯、二甲苯对 SOA 的形成贡献很大, SOA 产率分 别达到 $37 \%, 30 \%$ 和 $36 \%{ }^{[13]}$.

芳香烃化合物除了对 $\mathrm{O}_{3}$ 和 $\mathrm{SOA}$ 等二次污染物具有 显著贡献, 还与人体健康和气候变化息息相关 ${ }^{[14-17]}$. 1990 年, 美国联邦清洁空气法案修正案将苯、甲苯、乙 苯、二甲苯均列为有害空气污染物, 指出其会影响人体 血液生产、淋巴系统、生殖系统和中枢神经系统 ${ }^{[18-20]}$. 此外, 研究表明芳香烃在 $\mathrm{NO}_{x}$ 存在条件下可以氧化生成 硝基芳香烃类物质 ${ }^{[21]}$, 如硝基苯酚、二硝基苯酚、硝基 邻苯二酚和甲基硝基邻苯二酚等, 是棕色碳的关键吸光 物种，具有显著的气候效应 ${ }^{[22-23]}$.

鉴于芳香烃对大气污染、气候变化和人体健康的重 要作用, 近年来国内外研究者对其氧化机理进行了大量 的研究. 现阶段对芳香烃的氧化机理研究只能构建其大 致理论框架, 很多影响臭氧和二次有机气溶胶生成的关 键反应并未得到实验室研究的一致认可[24-29]。这就

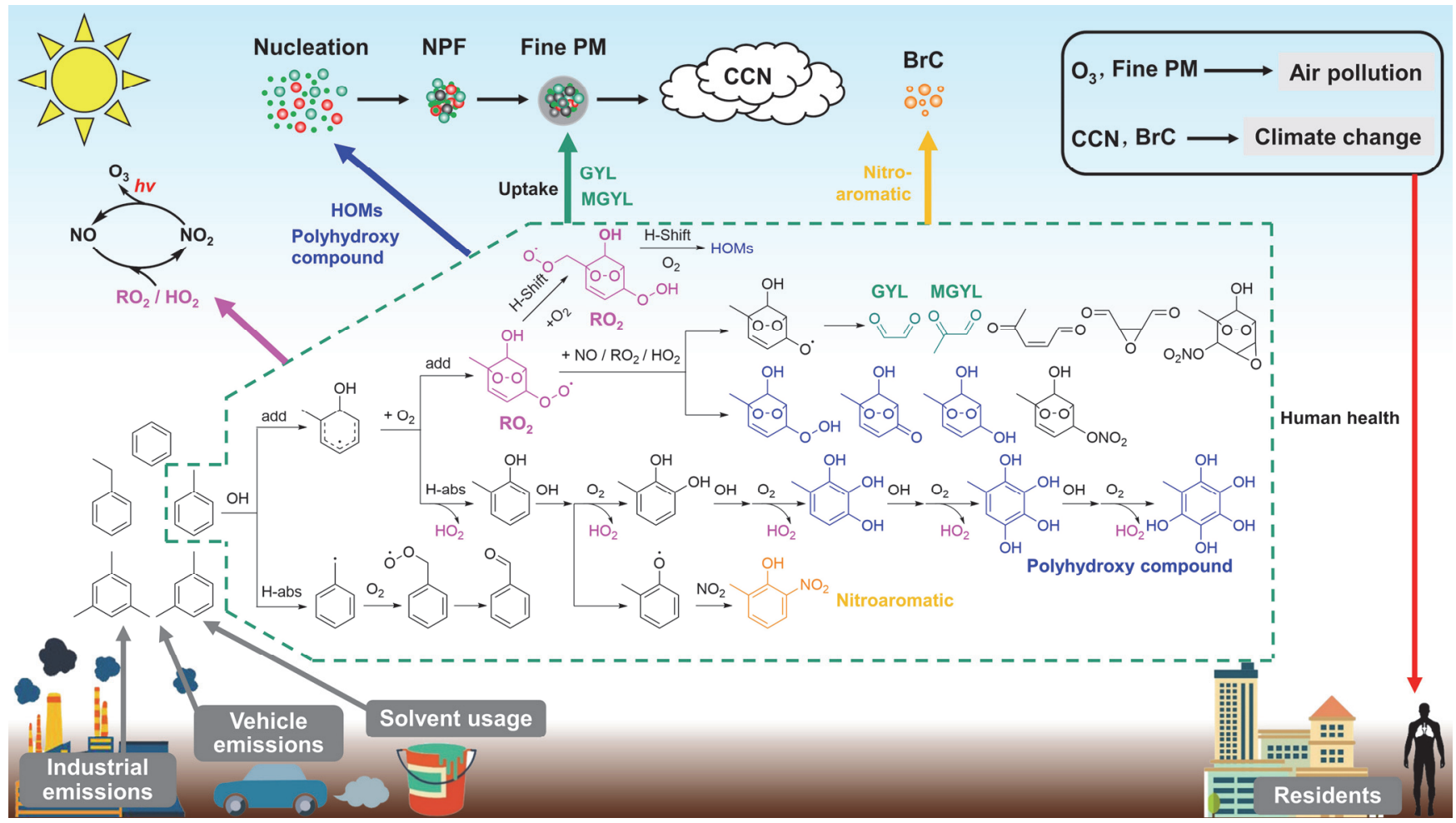

图 1 典型芳香烃氧化对空气质量、人体健康和气候的影响, abs 代表摘取反应, add 代表加成反应, HOMs 代表高含氧有机化合物; GYL 代表乙二 醛; MGYL 代表甲基乙二醛; NPF 代表新粒子生成; CCN 代表云凝结核; $\mathrm{BrC}$ 代表棕色碳

Figure 1 Effects of typical aromatic hydrocarbon oxidation on air quality, climate change and human health. abs: abstraction reaction; add: addition reaction; HOMs: highly oxygenated organic molecules; GYL: glyoxal; MGYL: methylglyoxal; NPF: new particle formation; CCN: cloud condensation nuclei; $\mathrm{BrC}$ : brown carbon 
导致了(1)不同研究或不同 $\mathrm{NO}_{x}$ 条件下，常见的中间氧 化产物的产率均存在较大的差异(图 2), 以甲苯为例, 其 中甲酚、乙二醛和甲基乙二醛的差异高达 5.9, 10.5 和 18.5 倍[25,30-50]; (2)国内外现有准特定化学机理(MCM)以 及高度归并的化学反应机理(加州大气污染研究中心机 理、碳键机理和区域大气化学机理)在进行芳香烃氧化 反应模拟时, 均存在不同程度高估产物的 $\mathrm{OH}$ 反应活 性、低估 $\mathrm{OH}$ 浓度、含氧挥发性有机物(OVOCs)产率和 $\mathrm{HO}_{2}$ 产率的现象; (3)基于现有芳香烃氧化机理, 显著低 估在高/低 $\mathrm{NO}_{x}$ 条件下的 $\mathrm{SOA}$ 形成 ${ }^{[13,51-52]}$.

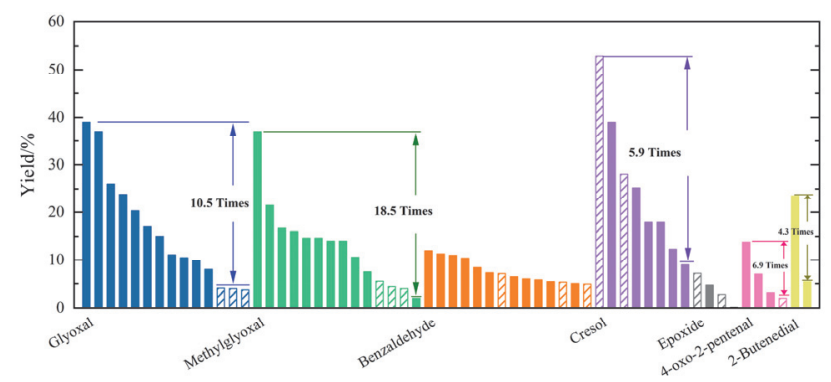

图 2 不同研究中甲苯氧化产物产率汇总 $[25,30-50]$, 实心柱代表有 $\mathrm{NO}_{x}$ 参与的甲苯氧化产物产率研究, 斜线柱代表没有 $\mathrm{NO}_{x}$ 参与的甲苯氧化 产物产率研究

Figure 2 Summary of yields of toluene oxidation products in different studies ${ }^{[25,30-50]}$. The solid box represents the product yield study with $\mathrm{NO}_{x}$ participation, while the slash box represents the product yield study without $\mathrm{NO}_{x}$ participation

综上所述, 芳香烃氧化机理及其环境效应的研究已 经成为当前大气环境化学领域最具挑战的研究之一. 本 文综述了芳香烃在大气中的主要氧化反应过程, 简要介 绍了芳香烃气相氧化机理研究方法和氧化中间产物的 主要测量技术, 重点关注芳香烃氧化反应研究中的新发 现和新理论.

\section{2 芳香烃的大气氧化反应}

芳香烃的大气氧化反应十分复杂, 一经排放到大气 中, 芳香烃类化合物苯环上的富电子结构就会迅速与大 气中的 $\mathrm{OH}$ 自由基反应, 苯环上的取代基则为 $\mathrm{OH}$ 自由 基的氢摘取反应提供了许多活性位点. 在大气的氧化过 程中，芳香烃氧化会产生多种含氧挥发性有机物 (OVOCs)及 $\mathrm{RO}_{2}$ 和 $\mathrm{HO}_{2}$ 自由基. 其中活性较高的 OVOCs, 能够与大气中的氧化剂 $\mathrm{OH}, \mathrm{O}_{3}$ 或者 $\mathrm{NO}_{3}$ 继续 反应, 通过氧化反应, 分子中氧原子个数增加, 形成挥 发性更低的氧化产物. 并将通过吸附、溶解等途径, 凝 结到大气中的细颗粒物中并使之增长, 是二次有机气溶 胶形成的重要途径 ${ }^{[53-55]}$. 而 $\mathrm{RO}_{2}$ 和 $\mathrm{HO}_{2}$ 自由基则可以参 与到 $\mathrm{NO}-\mathrm{NO}_{2}-\mathrm{O}_{3}$ 循环过程中, 与 $\mathrm{NO}$ 氧化成 $\mathrm{NO}_{2}$ 反应竞 争, 将 $\mathrm{NO}$ 转化为 $\mathrm{NO}_{2}$, 从而造成了臭氧的累积, 并导 致臭氧污染事件的发生. 自 20 世纪 70 年代, 科学家们 就已经开始了对芳香烃氧化机理的研究 ${ }^{[56]}$. 近 20 年来, 芳香烃氧化机理研究发展迅速, 但仍然存在很多争议与

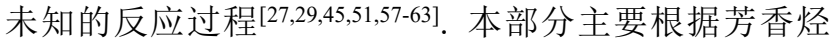
在大气中的氧化进程，逐步综述近年来对其氧化反应的 基本认识和最新进展. 由于甲苯是城市大气中含量最丰 富的芳香烃物种，且多项研究表明甲苯是对城市地区臭 氧和 SOA 贡献最大的芳香烃物种之一 ${ }^{[3,7,12]}$, 因此本研 究芳香烃氧化反应示意图主要以甲苯为例进行阐述.

\section{1 芳香烃的氧化引发反应}

芳香烃是大气中的活性物质，可以与 $\mathrm{OH}, \mathrm{Cl}, \mathrm{NO}_{3}$, $\mathrm{O}_{3}$ 等自由基发生反应 ${ }^{[4]}$. 在对流层, 芳香烃的转化主要 是通过光化学过程进行, 其中芳香烃主要与 $\mathrm{OH}$ (主要是 白天), $\mathrm{NO}_{3}$ (主要是夜间)和 $\mathrm{O}_{3}$ 自由基反应，芳香烃和 $\mathrm{OH}, \mathrm{NO}_{3}$ 以及 $\mathrm{O}_{3}$ 自由基的反应速率系数已被广泛地研 究 ${ }^{[4,59,64]}$, 反应系数如表 1 所示. 几乎所有的研究均表明 大气中芳香族化合物氧化的起始是由 $\mathrm{OH}$ 自由基的反应 主导的 ${ }^{[4,59,64]}$.

表 1 常见芳香烃化合物与 $\mathrm{OH}, \mathrm{NO}_{3}$ 和 $\mathrm{O}_{3}$ 自由基的反应速率 $[4,59,64]$

Table 1 The reaction rate of aromatic hydrocarbon compounds with $\mathrm{OH}, \mathrm{NO}_{3}$ and $\mathrm{O}_{3}$ radicals ${ }^{[4,59,64]}$

\begin{tabular}{|c|c|c|c|}
\hline Species & $\begin{array}{l}\text { Reaction with } \mathrm{OH} \\
\quad \times 10^{-12}\left(\mathrm{~cm}^{3} \bullet\right. \\
\left.\text { molecule }^{-1} \cdot \mathrm{s}^{-1}\right)\end{array}$ & $\begin{array}{c}\text { Reaction with } \\
\mathrm{NO}_{3} \\
\times 10^{-15}\left(\mathrm{~cm}^{3} \bullet\right. \\
\left.\text { molecule }^{-1} \cdot \mathrm{s}^{-1}\right)\end{array}$ & $\begin{array}{c}\text { Reaction with } \mathrm{O}_{3} \\
\times 10^{-20}\left(\mathrm{~cm}^{3} \bullet\right. \\
\left.\text { molecule }^{-1} \cdot \mathrm{s}^{-1}\right)\end{array}$ \\
\hline Benzene & 1.22 & $<0.03$ & \\
\hline Toluene & 5.63 & 0.07 & $<1$ \\
\hline Ethylbenzene & 7 & $<0.6$ & \\
\hline$o$-Xylene & 13.6 & 0.41 & \\
\hline$m$-Xylene & 23.1 & 0.26 & \\
\hline$p$-Xylene & 14.3 & 0.5 & \\
\hline $1,2,3-\mathrm{TMB}^{a}$ & 32.7 & 1.9 & \\
\hline $1,2,4-\mathrm{TMB}^{a}$ & 32.5 & 1.8 & \\
\hline $1,3,5-\mathrm{TMB}^{a}$ & 56.7 & 8.8 & \\
\hline
\end{tabular}

${ }^{a}$ TMB: Trimethylbenzene.

温度是影响芳香烃大气氧化初始反应的关键因素. 由温度依赖性动力学研究可知 ${ }^{[65]}$, 芳香烃和 $\mathrm{OH}$ 自由基 的反应有两条途径进行(图式 1), 从烷基取代基的碳氢 键或苯基中摘取 $\mathrm{H}$-原子, 或将 $\mathrm{OH}$ 自由基加成到芳香环 中形成羟基环己二烯基 (C-yl). 在常温常压下, $\mathrm{OH}$ 自由 基与芳香烃的加成途径占主导地位，而在温度高于 $350{ }^{\circ} \mathrm{C}$ 时, 会以氢摘取反应为主 ${ }^{[4,62,66]}$. 这主要是因为 加成反应是可逆的过程, 在高温条件下, $\mathrm{OH}$ 加成产物 会发生逆分解反应. 根据已有理论计算可知, 氢摘取反 应占比较小，甲苯摘氢产物产率一般为 $7 \% \sim 10 \%$, 二甲 苯摘氢反应产物产率一般为 $1 \% \sim 4 \%$. 目前普遍认为摘 氢反应是生成芳香醛类化合物的主要途径. 以甲苯为例 (图 3), 摘氢反应生成苯甲醛的途径(醛途径)占比 $\leqslant 10 \%$, 不同研究报道中甲苯氧化生成苯甲醛的产率范围为 $4.9 \% \sim 12 \%$, 这均表明了在实际大气情况下, $\mathrm{OH}$ 自由基 的加成主导了大气中芳香烃的氧化反应. Molina 等[67] (1999)通过化学电离质谱(chemical ionization mass spectrometer, CIMS, m/e 109)和 Bohn ${ }^{[68]}(2001)$ 通过 $308 \mathrm{~nm}$ 处的紫外激光吸收光谱仪器先后检测到甲苯的 $\mathrm{OH}$ 加成 
产物. 此外, 在加成反应中, 由于 $\mathrm{OH}$ 加入的位点具有 特异性, 分别可以发生本位 (ipso), 邻位 (ortho), 间位 (meta), 对位(para)加成, 得到的产物也大不相同(图式 $1)$.

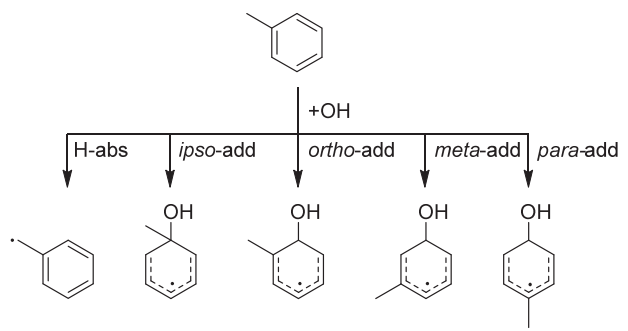

图式 1 甲苯的 $\mathrm{OH}$ 引发反应, abs 代表摘取反应, add 代表加成反应

Scheme 1 OH-Initiated oxidation reaction of toluene. abs: abstraction, add: addition

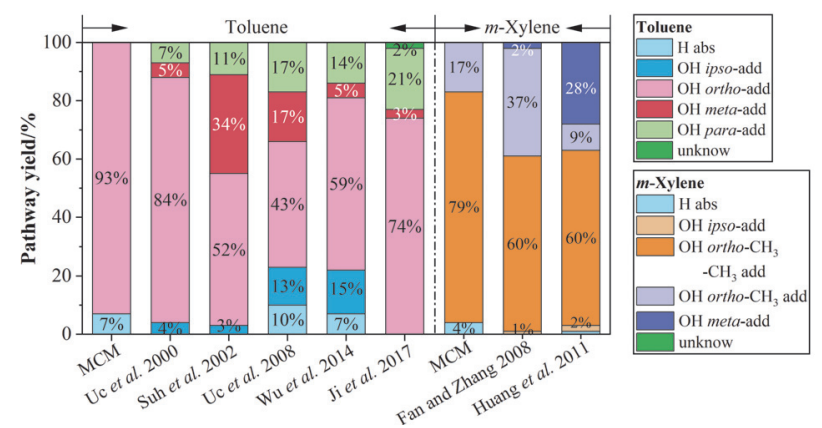

图 3 理论计算研究中芳香烃 $\mathrm{OH}$ 引发各反应位点产物产 率[38,57,62,66,69-71]

Figure 3 In the theoretical calculation study, literature yield of products initiated by $\mathrm{OH}-$ initiated oxidation reaction of aromatic hydrocarbon ${ }^{[38,57,62,66,69-71]}$
几乎所有的研究表明, 芳香烃苯环上的邻位加成反 应为主导反应，这主要是由于邻位加成的产物更加稳 定. 此外, 对位加成的产物由于具有相对较快的反应速 率, 也越发受到关注 ${ }^{[38,62]}$. Wang 等 ${ }^{[51]}$ 在甲苯氧化 $\mathrm{H}$ 转移 反应研究中提出对位加成产物在后续氢转移的反应过 程中的重要性. 整理近年来芳香烃 $\mathrm{OH}$ 引发反应各产物 产率的理论计算研究结果如图 3 所示. 可以看出甲苯的 邻位加成生成羟基环已二烯基为主导反应，产率达到 $43 \% \sim 93 \%$, 对位加成的产率约为 $7 \% \sim 21 \%{ }^{[38,57,62,66,69]}$. 对于间-二甲苯来说，与双甲基相邻的 $\mathrm{OH}$ 加成产物 (ortho $-\mathrm{CH}_{3}-\mathrm{CH}_{3}$ ) 占比最大, 达到 $60 \% \sim 70 \%$; 其次为单 甲基相邻的 $\mathrm{OH}$ 加成产物，占比达到 9\% 37\%，而间位 加成产物占比约为 $2 \% \sim 28 \%$. OH 加成反应作为芳香烃 氧化的主导途径, 其加成产物羟甲基环已二烯基(C-yl) 存在非芳香烃双键，具有更高的反应活性 ${ }^{[4]}$. C-yl 亦可 以通过摘氢和加成两种途径与 $\mathrm{O}_{2}$ 发生反应, 这就导致 了后续氧化过程的不同分支(图式 2).

\section{$2.2 \mathrm{C}-\mathrm{yl}$ 与 $\mathrm{O}_{2}$ 发生摘氢反应}

$\mathrm{C}-\mathrm{yl}$ 经摘氢途径可以形成环保留产物如甲酚 ${ }^{[61]}$ 和 $\mathrm{HO}_{2}$ 自由基, 是酚类产物生成的主导途径(酚途径). 芳 香烃常见加成产物形成酚途径的流程图如图式 2 所示.

近年来对酚途径的分支比存在较大的争议. 研究发 现，影响酚通道产率的关键因素为(1)产物的准确测量、 (2) $\mathrm{O}_{2}$ 浓度和(3) $\mathrm{NO}_{x}$ 浓度. 以甲苯为例, 不同实验室研

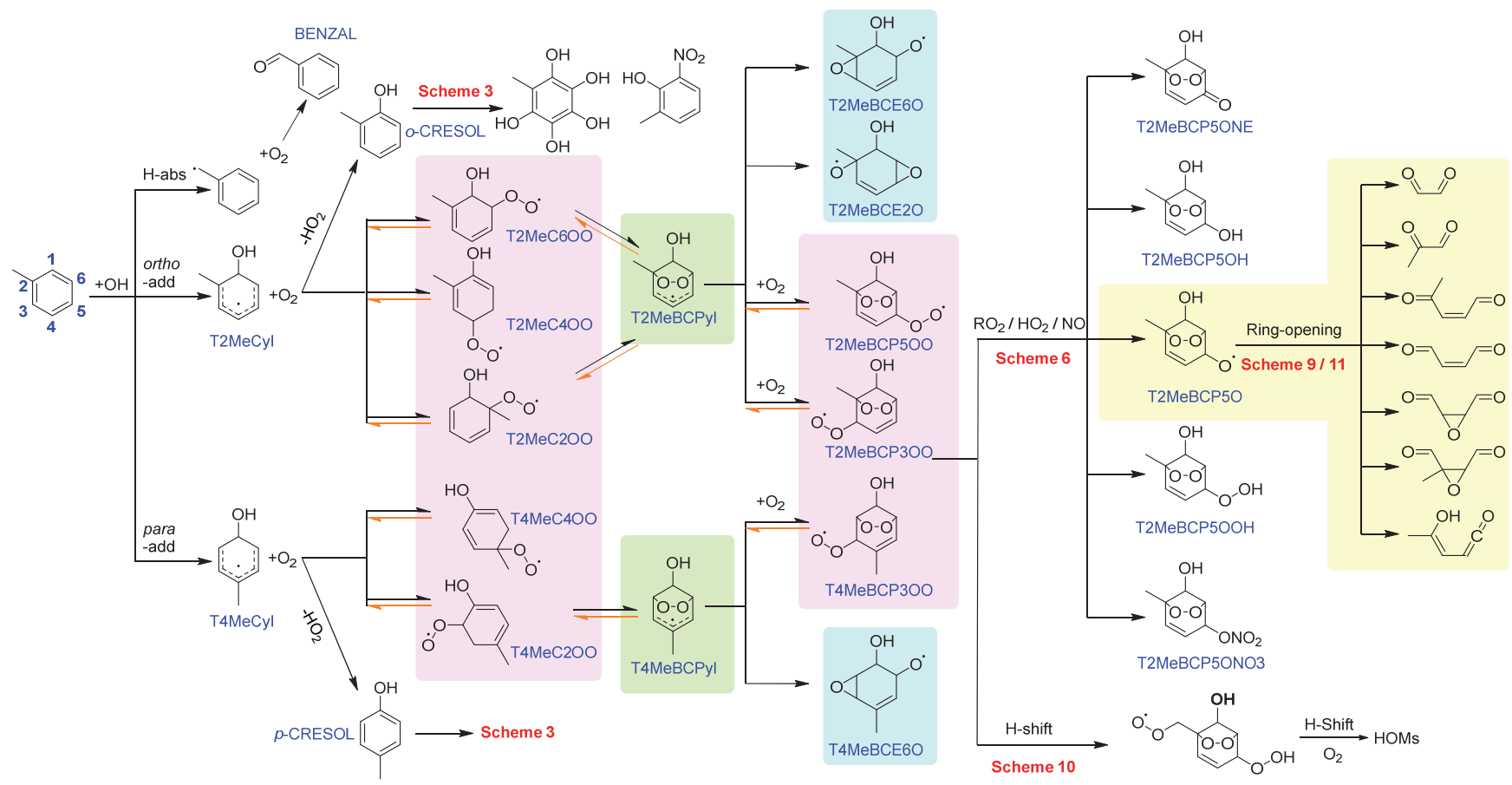

图式 2 芳香烃大气氧化主要反应途径(以甲苯为例), $\mathrm{T}$ 代表甲苯, $\mathrm{Me}$ 代表甲基, $\mathrm{yl}$ 代表碳基自由基, $\mathrm{B}$ 代表双环化合物, Tri 代表三环化合物, $\mathrm{C}$ 代 表羟基环已烷/龵基环已烯或羟基环己二烯, $\mathrm{P}$ 代表过氧桥, $\mathrm{E}$ 代表环氧桥, $\mathrm{O}$ 代表烷氧自由基, $\mathrm{OO}$ 代表过氧自由基

Scheme 2 The main reaction pathway of aromatic hydrocarbon oxidation (taking toluene as an example). T: Toluene, Me: Methyl, yl: Carbon-based radical, B: bicyclic compound, Tri: tricyclic compound, C: hydroxy cyclohexane/hydroxyl cyclohexene or hydroxyl cyclohexadiene, P: peroxide bridge, E: epoxide bridge, O: alkoxy radical, OO: peroxy radical 
究中甲苯氧化酚途径的占比结果差异较大, 范围从 $9.0 \%$ 到 $52.9 \%{ }^{[30-31,38,40,42-43]}$. 早期研究普遍认为酚途径 在氧化机理中的占比很小(约为 $10 \%$ ), $\mathrm{Ji}$ 等 ${ }^{[38]}$ 认为酚通 道在甲苯氧化中占比较大 $(39 \%)$, 而早期研究中使用测 量能力有限的气相色谱火焰离子检测仪(GC-FID)、气相 色谱质谱仪(GC-MS)或傅里叶变换红外光谱仪(FT-IR), 无法对酚途径的产物进行准确定量, 且在较早的烟雾箱 研究中, 未能考虑到甲酚与 $\mathrm{OH}$ 的多级后续反应, 导致 早期研究中计算的甲酚产率较低. 虽然 $\mathrm{Ji}$ 等研究解释了 早期甲酚产率低估的问题，但 Newland 等 ${ }^{[72]}$ 认为 $\mathrm{Ji}$ 等的 实验中使用的 $\mathrm{O}_{2}$ 浓度较小, 低于大气边界层中氧气浓 度的 $1 / 5000$, 使得反应向酚途径转移, 导致甲酚产率较 高. 部分研究和 $\mathrm{MCM}$ 机理趋向认为酚途径的产率为 $18 \%$ 左右 ${ }^{[39,43]}$. 此外, Birdsall 等 ${ }^{[34]}$ 认为 NO 浓度与苯氧 化体系中酚途径的分支比呈反相关, 是影响酚途径占比 变化的重要因素, 但值得注意的是除苯外, 其他芳香烃 均未表现出显著的 NO 依赖性.

由于甲酚 $\left(k_{\mathrm{OH}}=5.0 \times 10^{-11} \mathrm{~cm}^{3} \cdot \mathrm{molecule}{ }^{-1} \cdot \mathrm{s}^{-1}\right)$ 与 $\mathrm{OH}$ 的反应速率比其前体物甲苯 $\left(k_{\mathrm{OH}}=5.63 \times 10^{-12}\right.$ $\mathrm{cm}^{3} \cdot \mathrm{molecule}^{-1} \cdot \mathrm{s}^{-1}$ ) 快得多 [4], 甲酚与 $\mathrm{OH}$ 可以进一步发 生摘氢反应形成甲基苯氧基自由基，其后续可在高 $\mathrm{NO}_{x}$ 条件下与 $\mathrm{NO}_{2}$ 反应生成硝基酚类物质. Lin 等 ${ }^{[21]}$ 通过对 初始浓度为 $1205 \mu \mathrm{g} \cdot \mathrm{m}^{-3}$ 的甲苯进行高/低 $\mathrm{NO}_{x}$ 的烟雾箱 实验氧化研究, 发现高 $\mathrm{NO}_{x}$ 条件下甲苯氧化产生的硝基 芳香烃类物质是棕色碳的关键吸光物种，在 $300 \sim 500$ $\mathrm{nm}$ 波长范围内对棕色碳吸光贡献为 $40 \% \sim 60 \%$. 此外, 甲酚还能与 $\mathrm{OH}$ 发生加成反应形成二羟基甲苯或者双环 $\mathrm{RO}_{2}$ 自由基(图式 3). 其中，甲酚与 $\mathrm{OH}$ 加成形成二羟基 甲苯是甲酚主要的 $\mathrm{OH}$ 氧化途径，占比约为 $73 \%{ }^{[29,58]}$.

近年来，研究者们对于甲酚的后续反应有了更新的 认识 ${ }^{[29,38]}$. Schwantes 等 ${ }^{[29]}(2017)$ 对低 NO 和高 NO 条件 下甲苯与 $\mathrm{OH}$ 氧化反应进行了烟雾箱实验研究, 通过化 学电离质谱 (CIMS) 和高分辨实时直接分析质谱 (DART-MS)分别测量气相和颗粒相产物, 发现尽管气相 中甲酚途径分支比相对较低(20\%), 但甲酚的进一步与 $\mathrm{OH}$ 自由基反应可以形成氧化态更高、挥发性更低的产 物，对甲苯 SOA 的贡献很大 $(20 \% \sim 40 \%)$. Schwantes

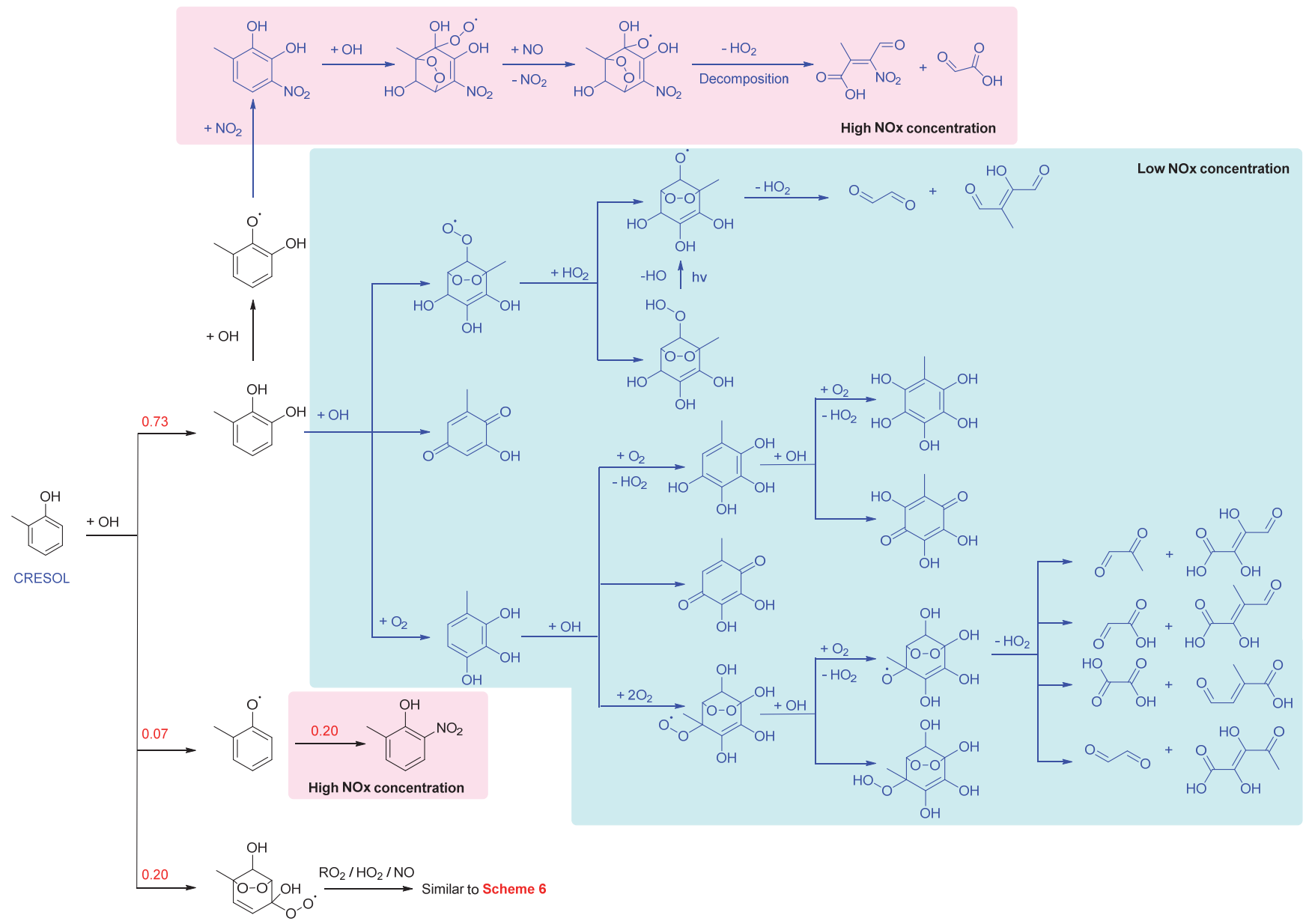

图式 3 甲酚在高低 $\mathrm{NO}_{x}$ 条件下与 $\mathrm{OH}$ 自由基反应机理 ${ }^{[29]} . \mathrm{MCM}$ 机制中包含的反应用黑色表示, Schwantes 等 ${ }^{[29]}$ 提出的反应用蓝色表示, 高 $\mathrm{NO}_{x}$ 条件下发生的反应用粉红色框框出, 低 $\mathrm{NO}_{x}$ 条件下发生的反应用蓝色框框出

Scheme 3 Reaction mechanism of cresol with $\mathrm{OH}$ radical under high and low $\mathrm{NO}_{x}$ conditions ${ }^{[29]}$. Reactions that have been included in the MCM mechanism are shown in black, reactions that proposed by Schwantes et al. ${ }^{[29]}$ are shown in blue, reactions that occur under high $\mathrm{NO}_{x}$ conditions are shown in pink box, and reactions that occur under low $\mathrm{NO}_{x}$ conditions are shown in blue box 
等[29]指出, 无论低 $\mathrm{NO}\left(0 \mu \mathrm{g} \cdot \mathrm{m}^{-3}\right)$ 还是高 $\mathrm{NO}$ 条件 $(86$ $\left.98 \mu \mathrm{g} \cdot \mathrm{m}^{-3}\right)$ 下，二羟基甲苯均可以与 $\mathrm{OH}$ 自由基发生多 级加成反应, 形成三羟基甲苯、四羟基甲苯或二羟基甲基苯醌、三羟基-甲基苯醌，以及可能来自双环中间体 途径的各种开环产物. 在高 $\mathrm{NO}$ 条件下 $\left(86 \sim 98 \mu \mathrm{g} \cdot \mathrm{m}^{-3}\right)$, 二羟基甲苯与 $\mathrm{OH}$ 自由基则会发生摘氢反应，随后与 $\mathrm{NO}_{2}$ 反应生成二羟基硝基甲苯. 但是由于气态和颗粒态 的二羟基硝基甲苯含量较低, 表明氢摘取途径不是二羟 基甲苯 $\mathrm{OH}$ 氧化的主要途径. 甲酚与 $\mathrm{OH}$ 自由基后续反 应途径如图式 3 所示, 这里将甲酚与 $\mathrm{OH}$ 自由基发生多 级加成反应称为多差基化合物形成机理, 暂未被纳入到 成型的 MCM 机理和区域大气化学机理(RACM)中. 而 对其他芳香烃的多着基机理暂无研究发表, 其反应可以 使用 Schwantes 等的理论进行类推预测.

综上所述, 甲酚进一步与 $\mathrm{OH}$ 自由基氧化反应可以 形成 $\mathrm{HO}_{2} 、 \mathrm{RO}_{2}$ 以及多酚物质, 其中 $\mathrm{HO}_{2} 、 \mathrm{RO}_{2}$ 可以通 过将 $\mathrm{NO}$ 转化为 $\mathrm{NO}_{2}$ 来促进臭氧的产生, 而多酚物质氧 化态更高、挥发性更低很容易通过气粒分配形成 SOA, 且其对甲苯产生的 SOA 的贡献占比可达 $20 \% \sim 40 \%{ }^{[29]}$.

\section{$2.3 \mathrm{C}-\mathrm{yl}$ 与 $\mathrm{O}_{2}$ 的多代加成反应}

$\mathrm{C}-\mathrm{yl}$ 除了与 $\mathrm{O}_{2}$ 发生摘氢反应外, 还可以和 $\mathrm{O}_{2}$ 发生 多代加成反应, 形成一系列 $\mathrm{RO}_{2}$ 自由基 ${ }^{[62,73-74]}$.

\subsection{1一代加氧反应}

$\mathrm{C}-\mathrm{yl}$ 在第一个 $\mathrm{O}_{2}$ 加入后主要可以形成初级过氧自 由基(C-OO). 1995 年, Bartolotti 等[75]通过理论预测了甲 苯氧化过程中存在稳定的环氧中间体和产物的可能性, 根据理论计算得出双环己烯环氧烷氧基(BCE-O)在能量 上比同分异构体环己烯过氧基(C-OO)或过氧桥联双环 氧基(BCP-yl)更有利. 两年后, $\mathrm{Yu}$ 等[76]通过红外光谱实 验虽证实了 BCE-O 自由基的形成, 但发现在 C-yl 和 $\mathrm{O}_{2}$ 加成反应中直接生成环氧化物具有很高的能量壁 垒 ${ }^{[77-79]}$, 而形成 C-OO 的途径更可行 ${ }^{[80]} .1999$ 年, C-OO 在 Molina 等 ${ }^{67]}$ 的研究中首次被 CIMS 检测到, 证实了这 一反应的发生.

图式 2 显示了甲苯氧化生成的烷基自由基第一次加 $\mathrm{O}_{2}$ 的反应的流程图. C-yl 与 $\mathrm{O}_{2}$ 加成反应具有很强的位置 特异性, 这对产物的形成有很大的影响 ${ }^{[62]}$. 理论研究发 现 C-yl 与 $\mathrm{O}_{2}$ 加成产物的形成主要是在邻位添加 $\mathrm{O}_{2}{ }^{[63]}$. 通过拟合 $\mathrm{OH}$ 自由基和 $\mathrm{C}-\mathrm{yl}$ 加合物的紫外吸收光谱, Bohn ${ }^{[68]}$ 发现这种加成反应是可逆的.

影响芳香烃氧化烷基自由基 $(\mathrm{C}-\mathrm{yl})$ 与 $\mathrm{O}_{2}$ 第一次加成 的主要因素为 $\mathrm{NO}_{x}$ 的浓度. Koch 等 ${ }^{[81]}$ 发现 $\mathrm{C}-\mathrm{yl}$ 与 $\mathrm{O}_{2}$ 、 $\mathrm{NO}_{x}$ 和 $\mathrm{HO}_{x}$ 等自由基均可能发生反应. Koch 等研究了 $\mathrm{C}-\mathrm{yl}$ 与 $\mathrm{O}_{2} 、 \mathrm{NO}$ 和 $\mathrm{NO}_{2}$ 的反应, 得到速率常数分别为 $(5.6$ $\pm 1.5) \times 10^{-16}(299 \mathrm{~K}),<3.0 \times 10^{-14}(333 \mathrm{~K})$ 和 $(3.6 \pm 0.5)$ $\times 10^{-11} \mathrm{~cm}^{3} \cdot$ molecule ${ }^{-1} \cdot \mathrm{s}^{-1}(300 \mathrm{~K})$, 这表明 $\mathrm{C}$-yl 在 $\mathrm{NO}_{x}$ 为 $\mu \mathrm{g} \cdot \mathrm{m}^{-3}$ 量级的典型环境浓度下只与 $\mathrm{O}_{2}$ 反应. Nishino 等 ${ }^{411}$ 在实验室研究发现, 当 $\mathrm{NO}_{2}$ 浓度达到极高的 6207 $\mu \mathrm{g} \cdot \mathrm{m}^{-3}$ 时, $\mathrm{C}-\mathrm{yl}$ 与 $\mathrm{NO}_{2}$ 的反应也同样重要.

2.3.2 二代加氧反应

初级过氧自由基(C-OO)的进一步氧化是在环化形 成过氧桥双环自由基( $\mathrm{BCP}-\mathrm{yl}$ ) 和与 $\mathrm{RO}_{2}, \mathrm{HO}_{2}$ 以及 $\mathrm{NO}$ 反 应形成烷氧自由基 $(\mathrm{C}-\mathrm{O})$ 等产物之间的竞争决定的.

(1)环聚反应形成过氧桥双环自由基(BCP-yl)

芳香烃氧化过程存在两类典型的环聚反应，第一类 过氧自由基环化形成过氧桥化合物，第二类是烷氧基环 化形成环氧桥化合物. 初级过氧自由基可以迅速发生第 一类闭环反应，形成过氧桥双环自由基 BCP-yl (图式 2). 此外, C-OO 环聚形成 BCP-yl 的反应还被证实为可逆反

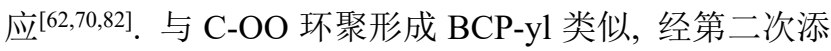
加 $\mathrm{O}_{2}$ 后的双环过氧自由基(BCP-OO)也可能发生同样的 环聚反应，形成过氧桥三环自由基(TriCPP-yl), 如图式 4 所示. 仅有少数理论研究表明芳香烃氧化过程中会有 此反应的发生 ${ }^{[62,83]}$, 且反应壁垒很高, 一般难以形成, 现无实验室研究验证.

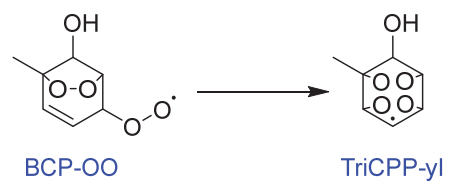

图式 4 双环 $\mathrm{RO}_{2}$ 环聚反应形成三环过氧桥烷基自由基流程图 Scheme 4 Tricyclic peroxy bridge carbon-based radical formed by bicyclic $\mathrm{RO}_{2}$ cyclic polymerization reaction

(2) BCP-yl 与氧气加成反应

过氧桥双环自由基 $\mathrm{BCP}-\mathrm{yl}$ 的后续氧化是由其与 $\mathrm{O}_{2}$ 二次加成反应形成双环过氧自由基(BCP-OO)和断过氧 桥重排形成环氧桥烷氧自由基(BCE-O)之间的竞争决定 的.

根据烷基自由基容易与 $\mathrm{O}_{2}$ 发生加成反应的特征, BCP-yl 可继续与 $\mathrm{O}_{2}$ 发生加成反应, 形成 BCP-OO 自由 基以避免过氧桥的重新打开 ${ }^{[73]}$. 理论计算认为二次以 上的加氧过程产物更稳定, 其再解离速率系数很低, 一 般很难进行逆反应 ${ }^{[63]}$. BCP-OO 自由基已经被 Birdsall 等[60]在 2010 年使用 CIMS 首次直接观测到. 芳香烃氧化 中主要的环裂解途径是在 BCP-O 自由基中的环断裂反

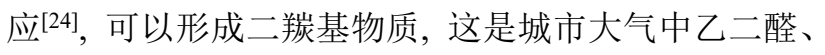
甲基乙二醛的主要二次来源 ${ }^{[84]}$. BCP-yl 自由基二次加 $\mathrm{O}_{2}$ 反应的研究相对较少, 现有理论研究表明其反式加 成更为有利. 甲苯氧化过程中 $\mathrm{BCP}-\mathrm{yl}$ 与 $\mathrm{O}_{2}$ 加成反应如 图式 2 所示. 由于 BCP-OO 环聚反应形成 TriCPP-yl 的 能垒很大, 因此 $\mathrm{BCP}-\mathrm{OO}$ 主要与 $\mathrm{RO}_{2}, \mathrm{HO}_{2}$ 和 $\mathrm{NO}$ 反应生 成烷氧自由基(BCP-O), 过氧化合物(BCP-OOH), 醌类 化合物 $(\mathrm{BCP}=\mathrm{O})$, 差基化合物 $(\mathrm{BCP}-\mathrm{OH})$ 等, 此反应在 第 2.4 节进行详细描述.

(3) BCP-yl 过氧桥断裂重排形成环氧桥烷氧自由基 (BCE-O)

BCP-yl 除了和 $\mathrm{O}_{2}$ 发生加成反应外, 还可通过断过 
氧桥并进行分子内重排, 形成环氧桥烷氧自由基 (BCE-O), 反应过程如图式 2 所示. 理论研究认为此途 径具有较大的反应壁垒, 反应速率较低, 一般无法竞争 过与 $\mathrm{O}_{2}$ 的二次加成反应.

\subsection{3 三代加氧反应}

二次加氧形成的双环过氧化物(BCP-OO) 可以通过 和 $\mathrm{NO}, \mathrm{HO}_{2}$ 与 $\mathrm{RO}_{2}$ 等自由基反应形成双环烷氧化合物 (BCP-O), 而 BCP-O 通过烷氧自由基环氧化则可以形成 三环烷基自由基(TriCPE-yl). 同样地, TriCPE-yl 也可以 发生与 $\mathrm{O}_{2}$ 的加成反应, 这就是芳香烃氧化过程中的第 三次加氧途径(图式 5). Wang 等估计, 在苯氧化生成的 TriCPE-yl 上加成 $\mathrm{O}_{2}$ 的反应速率系数在 $10^{-11} \mathrm{~cm}^{3}$ • molecule ${ }^{-1} \cdot \mathrm{s}^{-1}$ 左右, 近似于环已基与 $\mathrm{O}_{2}$ 加成的速率系

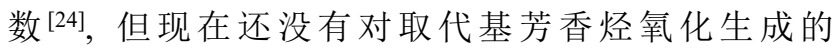
TriCPE-yl 与 $\mathrm{O}_{2}$ 加成的速率系数的实验室研究.

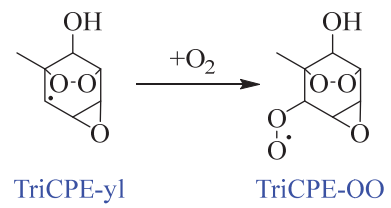

图式 5 TriCPE-yl 自由基与 $\mathrm{O}_{2}$ 的加成反应

Scheme 5 The addition reaction of TriCPE-yl radical with $\mathrm{O}_{2}$

\section{$2.4 \mathrm{RO}_{2}$ 与 $\mathrm{NO}, \mathrm{HO}_{2}, \mathrm{RO}_{2}$ 的双分子反应}

如前所述, 芳香烃氧化产生的烷基自由基与氧气加 成可以形成多种类型的 $\mathrm{RO}_{2}$ 自由基, 包括单环过氧自由 基 C-OO, 双环过氧自由基 BCP-OO 和三环过氧自由基 TCPE-OO. 在对流层条件下, $\mathrm{RO}_{2}$ 自由基由于较高的活 性, 存在很多反应途径. 这些反应包括一系列的双分子 反应, 即 $\mathrm{RO}_{2}$ 与 $\mathrm{NO}, \mathrm{NO}_{2}, \mathrm{NO}_{3}, \mathrm{HO}_{2}$, 以及与大气中其他 $\mathrm{RO}_{2}$ 自由基的自反应和交叉反应，这些反应相互之间存 在竞争关系. 对于某些类别的 $\mathrm{RO}_{2}$ 还可能发生单分子异 构化反应，如 $\mathrm{H}$ 转移反应或环聚反应.

$\mathrm{RO}_{2}$ 的后续反应途径受 $\mathrm{NO}_{x}$ 浓度影响较大. 一般在 高 $\mathrm{NO}_{x}$ 环境中, $\mathrm{RO}_{2}$ 与 $\mathrm{NO}$ 反应将其氧化为 $\mathrm{NO}_{2}$ (图式 $6 \mathrm{a})$, 对 $\mathrm{O}_{3}$ 的形成起关键作用, 该反应是污染条件下 $\mathrm{RO}_{2}$ 自由基的主要反应. $\mathrm{RO}_{2}$ 与 $\mathrm{NO}$ 反应的终止通道为形 成有机硝酸酯产物 $\mathrm{RONO}_{2}$ 的反应分支, 这一分支比的 贡献取决于 $\mathrm{RO}_{2}$ 的结构和大小. 当 $\mathrm{RO}_{2}$ 自由基与 $\mathrm{NO}_{2}$ 反应时, 可以形成稳定的过氧硝酸酯产物 $\mathrm{ROONO}_{2}{ }^{[85]}$. 而 $\mathrm{RO}_{2}$ 自由基与 $\mathrm{NO}_{3}$ 的反应主要发生在夜间, 提供了可 能补充夜间链氧化过程的自由基传播途径 ${ }^{[86]}$.

在 $\mathrm{NO}_{x}$ 浓度较低的环境中, $\mathrm{RO}_{2}$ 与 $\mathrm{HO}_{2}$ 和 $\mathrm{RO}_{2}$ 自由 基的反应变得更为重要. $\mathrm{RO}_{2}$ 与 $\mathrm{HO}_{2}$ 的反应过程如图式 $6 \mathrm{~b}$ 所示, 产物主要为烷氧自由基 (RO) 和过氧化物 $(\mathrm{ROOH})$, 其中 $\mathrm{ROOH}$ 占主导. $\mathrm{Ng}$ 等 ${ }^{[13]}$ 认为低 $\mathrm{NO}_{x}$ 条件 下, $\mathrm{RO}_{2}$ 与 $\mathrm{HO}_{2}$ 反应生成的 $\mathrm{ROOH}$ 对 $\mathrm{SOA}$ 具有重要贡 献. $\mathrm{RO}_{2}$ 与 $\mathrm{RO}_{2}$ 的反应过程如图式 $6 \mathrm{c}$ 所示, 产物主要为
烷氧自由基 $(\mathrm{RO})$ 、醌类化合物 $(\mathrm{R}=\mathrm{O})$ 和羟基化合物 $(\mathrm{ROH})$ ，其中 $\mathrm{RO}$ 占主导. 虽然 $\mathrm{RO}_{2}$ 与 $\mathrm{HO}_{2} 、 \mathrm{RO}_{2}$ 自由基 的反应可以通过与 $\mathrm{NO}$ 反应之间的竞争而抑制 $\mathrm{O}_{3}$ 的形 成，但产生的 RO 和 $\mathrm{ROOH}$ 也对大气自由基的浓度和 $\mathrm{SOA}$ 的生成有重要影响. 此外, 在 $\mathrm{NO}_{x}$ 浓度较低的条件 下，双环 $\mathrm{RO}_{2}$ 自由基会发生单分子异构化(过氧基环化 形成过氧桥/ $\mathrm{H}$ 转移反应)反应与双分子反应竞争. 这些 反应在 $\mathrm{HO}_{x}$ 自由基循环以及产生高氧化态多官能团有 机物(HOMs) 的快速链氧化机制中发挥重要作用 ${ }^{[29,51,87]}$.

综上, $\mathrm{RO}_{2}$ 各类双分子反应的反应速率和分支比会 显著影响后续氧化产物的分布和官能团类型, 以及它们 的物理化学性质(例如挥发性和溶解性), 进而影响臭氧 和 SOA 生成 ${ }^{[88]}$. 芳香烃的氧化会产生大量 $\mathrm{RO}_{2}$ 自由基, 难以对每个反应进行详细描述, 因此建立简化的参数化 方法是非常必要的. Jenkin 等基于对大量的 $\mathrm{RO}_{2}$ 自由基 与 $\mathrm{NO}, \mathrm{NO}_{2}, \mathrm{NO}_{3}, \mathrm{HO}_{2}, \mathrm{RO}_{2}$ 反应的研究构建数据 集 ${ }^{[89-116]}$, 采用参数化的方法定量 $\mathrm{RO}_{2}$ 各类双分子反应 的反应速率和分支比. 此方法在准特定机理 MCM 中得 到了广泛的应用. 根据 Jenkin 等 ${ }^{[88]}$ 的参数化研究发现, 对于某个特定 $\mathrm{RO}_{2}$ 自由基, 其后续反应速率取决于 $\mathrm{RO}_{2}$ 的结构(官能团的类型)和环境条件(压力和温度).

\section{5 烷氧自由基环氧化反应}

Andino 等 ${ }^{[117]}$ 和 Suh 等 ${ }^{[118]}$ 的理论研究发现, 芳香烃 氧化生成的双环烷氧自由基(BCP-O)会发生环裂解反 应，形成乙二醛(GYL)、甲基乙二醛(MGYL)和不饱和 1,4-二羰基副产物. 且 Suh 等通过计算表明这些双环烷 氧基应产生等量的 GYL/MGYL 及其副产物. 但是在实 验室模拟中测得的 GYL 和 MGYL 的总产率远高于副产 物的总产率 $[25,37,119]$. 为了折中模型预测和实验观测之间 的差异, 以甲苯为例, $\mathrm{MCM}$ 机理将甲苯氧化双环烷氧 自由基的环断裂途径中 MGYL 的副产物 $\mathrm{C}_{4} \mathrm{H}_{4} \mathrm{O}_{2}$ 等分为 丁烯二醛和 2(5H)-呋喃酮(丁烯二醛的异构体), 将 GYL 的 $\mathrm{C}_{5} \mathrm{H}_{6} \mathrm{O}_{2}$ 副产物等分为 1,4-二羰基化合物和甲基2(5H)-呋喃酮(1,4-二羰基化合物的异构体 ${ }^{[62]}$. 然而, 尽 管甲基-2(5H)-呋喃酮还可能由 1,4-二羰基化合物的光解 形成, 但 Smith 等 ${ }^{[43]}$ 在甲苯氧化烟雾箱实验中, 仅有微 量的呋喃酮仅被检测出来. Wang 等 ${ }^{[24]} 2013$ 年在对苯 的氧化机理研究中通过理论计算提出一种新的烷氧自 由基环氧化的机理, 新的机制表明乙二醛的产率是丁烯 二醛的三倍, 这与以往实验测量结果一致 ${ }^{[25,37,119]}$. $\mathrm{Wu}$ 等 ${ }^{[62]}$ 和 Pan 等 ${ }^{[82}$ 通过理论计算分别发现甲苯和二甲苯 氧化中也同样存在烷氧自由基环氧化的可行性. 但是这 一新机理还需要对其产物环氧化物进行验证. 2019 年, Zaytsev 等[26]报道了使用 CIMS 在烟雾箱实验中检测到 烷氧自由基环氧化的产物环氧物种，实现了对该机制的 验证. 

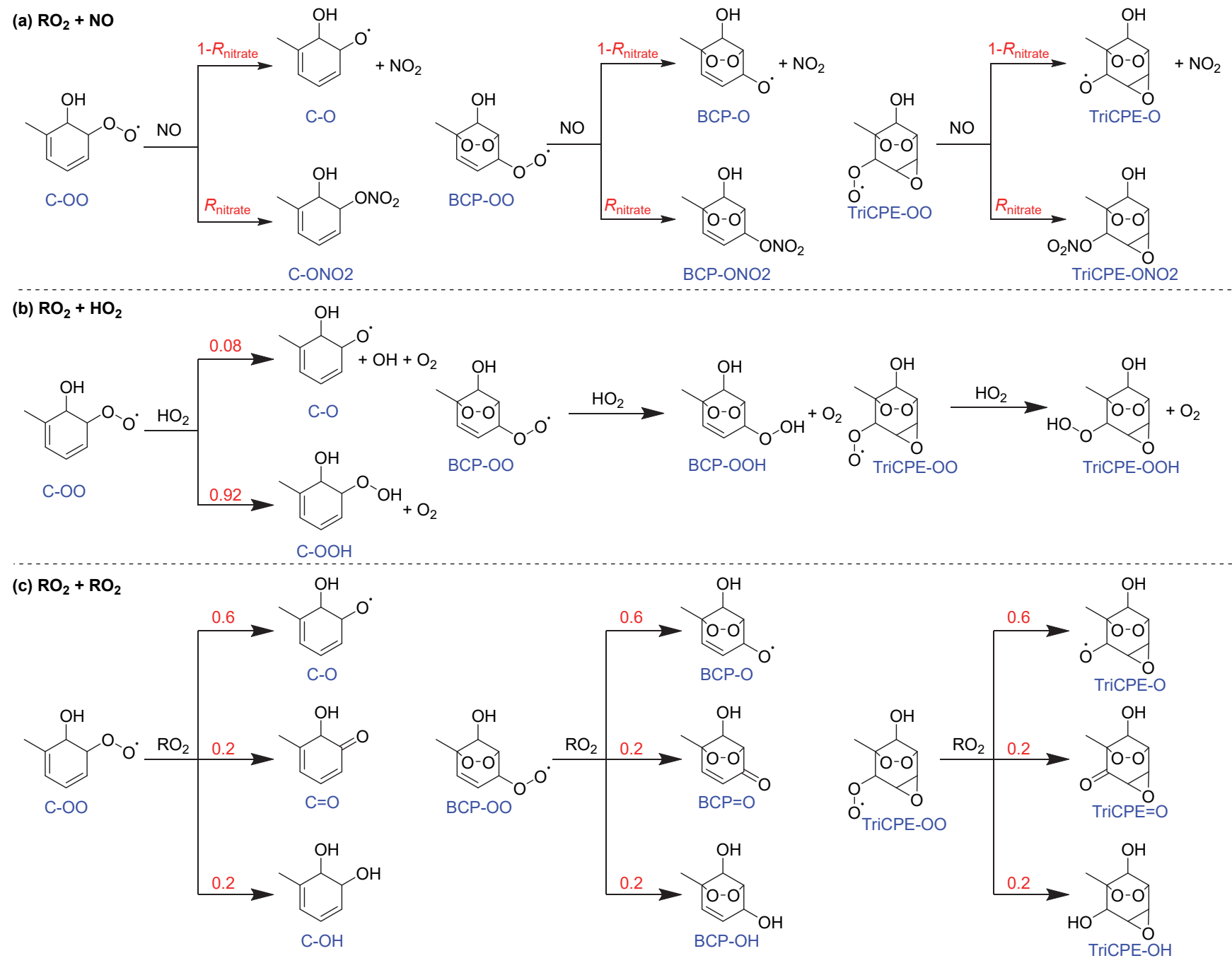

图式 $6 \mathrm{RO}_{2}$ 与 $\mathrm{NO}, \mathrm{HO}_{2}$ 和 $\mathrm{RO}_{2}$ 的双分子反应. $R_{\mathrm{nitrat}}$ 代表 $\mathrm{RO}_{2}$ 与 $\mathrm{NO}$ 反应生成的有机硝酸酯产物通道的分支比. 图式 6 中各反应的分支比参照了 Jenkin 等 ${ }^{[88}$ 的研究

Scheme 6 Bimolecular reactions of $\mathrm{RO}_{2}$ with $\mathrm{NO}, \mathrm{HO}_{2}$ and $\mathrm{RO}_{2}$. $R_{\text {nitrate }}$ represent the bifurcation ratio of organic nitrate product channels produced by reaction of $\mathrm{RO}_{2}$ with NO. The branching ratio of each reaction in Scheme 6 refers to the study by Jenkin et al. ${ }^{[88]}$

芳香烃氧化过程中存在着三种典型的烷氧自由基, 包括单环烷氧基(C-O)、双环过氧桥烷氧基(BCP-O)和双 环环氧桥烷氧基(BCE-O), 这些烷氧自由基的环氧化反 应主要如图式 7 所示, 是芳香烃氧化过程中第二类典型 的环聚反应. 研究表明, 这些环氧化反应是可逆的, 并 与其他烷氧自由基损失过程相互竞争.

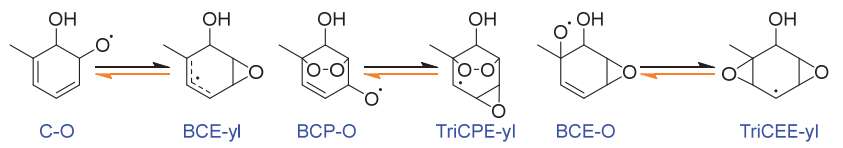

图式 7 芳香烃氧化中间产物烷氧自由基环氧化反应

Scheme 7 Aromatic hydrocarbon oxidation intermediate product alkoxy radical epoxidation reaction

单环烷氧基(C-O)的环氧化研究较少, 现有理论计 算认为此反应的能垒很小 ${ }^{[78,83]}$. 而双环烷氧基的研究更 为详细, Wang 等 ${ }^{[24]}$ 和 $\mathrm{Wu}$ 等 ${ }^{[62]}$ 认为双环烷氧基的环氧化
对产物的分布影响更大．考虑到双环过氧桥烷氧基 (BCP-O) 的环氧化与芳香环断裂竞争, 如前所述可解决 乙二醛/甲基乙二醛与其副产物产率不匹配的情况. 对 于双环环氧桥烷氧自由基(BCE-O)来说, 其环氧化与开 环反应也同样存在着竞争. BCE-O 的开环可以形成环氧 化物. MCM 认为 BCE-O 的开环反应是芳烃氧化产生环 氧化物的主要途径, 占比达到 10\% (图式 8, P1 通道), 但 现阶段实验室研究均发现 MCM 高估了这一途径, 提出 此途径的实际产率小于 $1 \%{ }^{[26]}$. Wang 等 ${ }^{[24]}$ 在对苯的氧化 机理研究中提出 BCE-O 的环氧化反应可以解释上述环 氧化物产率较低的情况(图式 $8, \mathrm{P} 2$ 通道). 通过估算图式 8 中各通道 $(\mathrm{P} 1 \sim \mathrm{P} 4)$ 反应速率, 发现 BCE-O 几乎都是通 过环氧化通道 P2 来去除的, 随后经 P3 和 P4 两通道分 别生成环保留和环裂解产物. 但此反应途径目前仅在苯 的氧化研究中提到. 

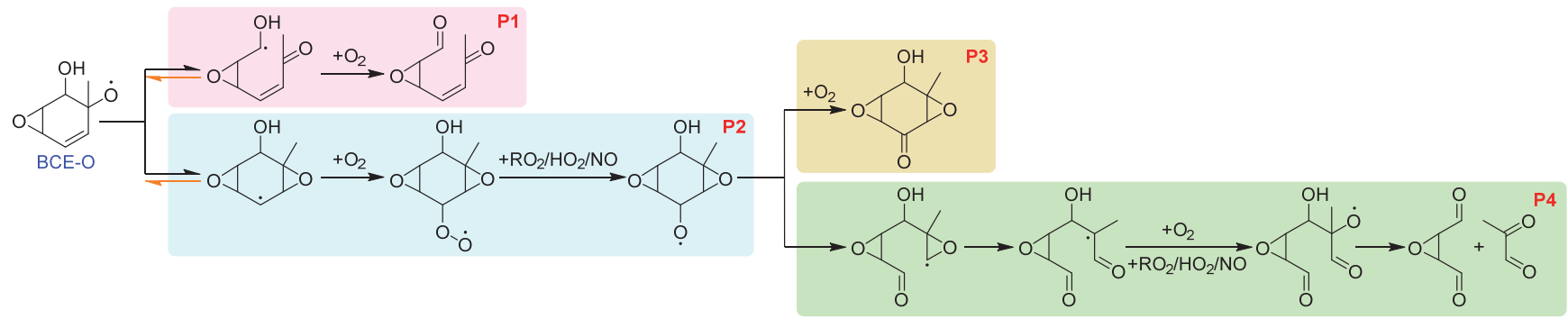

图式 8 芳香烃氧化过程环氧化物形成过程 ${ }^{[23,58]}$

Scheme 8 Fates of epoxides formation during aromatic hydrocarbon oxidation mechanism ${ }^{[23,58]}$

\section{6 环断裂反应}

烷氧自由基( $\mathrm{RO})$ 中 $\beta-\mathrm{C}-\mathrm{C}$ 键断裂是芳香烃大气氧 化过程中碳链断裂的主要途径. $\mathrm{C}-\mathrm{O}$ 一般会断开 $\mathrm{HOC}-\mathrm{CO}$ •键, 且该反应通常在 $\mathrm{NO}$ 浓度很高的情况下 发生, 否则 $\mathrm{C}-\mathrm{O}$ 环断裂反应无法与 $\mathrm{C}-\mathrm{O}$ 环聚形成 BCE-yl 反应竞争. 芳香烃氧化的主要碎裂途径是双环 烷氧自由基(BCP-O) 的开环, 其开环主要是断开 $\mathrm{O}-\mathrm{O}-\mathrm{C}-\mathrm{C}-\mathrm{O} \bullet$ 中的 C-C 键. 现有的 BCP-O环断裂反应包 含三种版本, 如图式 9 所示. 经典的环断裂反应折中模

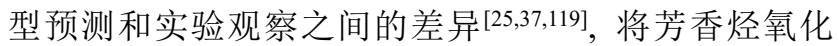
BCP-O 的环断裂途径中小分子二羰基醛类(如乙二醛、 甲基乙二醛和乙基乙二醛等)的副产物等分为烯醛类化 合物与呋喃酮类化合物(图式 9(a)). 第二种 BCP-O 的环 断裂途径引入 Wang 等 ${ }^{[24]} 2013$ 年提出一种新的烷氧自由 基环氧化的机理, 通过引入环氧化合物来解释实验和理 论计算中的差异. 这一理论在对甲苯和二甲苯的研究中 也得以证实 ${ }^{[24,62,74]}$, 根据这一理论推测出甲苯氧化过程 BCP-O 环断裂反应过程如图式 9(b)所示.
但由于开环产物中的多羰基化合物异构体种类多、 活性较高, 它们的测量受限于仪器的定性定量能力及反 应产物停留时间等, 且较少有研究可检测到环氧-二羰 基物质(epoxide)的存在, 现有研究对初级氧化反应生成 的二羰基化合物反应产率存在较大争议, 已有研究中乙 二醛的产率范围为 $3.9 \% \sim 39 \%$, 甲基乙二醛的产率范围 为 $2 \% \sim 37 \%$, 环氧-二羰基产物的产率范围为 $4.7 \%$ $7.2 \%$ [25,31-33,36-37,41,43-45]. 最新研究发现, BCP-O 自由基发 生环氧闭环反应生成环氧羰基化物的途径并不重要, 并 提出了 BCP-O 环断裂后的醛类 $\mathrm{H}$ 转移新理论, 这部分 将在 2.7 节进行详细讨论. 第三种 BCP-O 的环断裂引入 醛类 $\mathrm{H}$ 转移新理论，如图式 9 (c) 所示.

\section{$2.7 \mathrm{H}$ 转移反应}

近年来, 由于 $\mathrm{RO}_{2}$ 自由基分子内氢转移反应途径成 功解释了异戊二烯 $\mathrm{OH}$ 氧化过程、并揭示存在更有效的 $\mathrm{OH}$ 再生反应机制[120-121], 芳香烃氧化过程中的 $\mathrm{H}$ 转移 反应也越来越受到重视. 至今, 研究者们通过量子化学 计算先后提出了两种 $\mathrm{H}$ 转移的新理论, 分别是(1)双环 过氧自由基氢转移理论 ${ }^{[51]}$ 和(2)醛类化合物氢转移理 论 $^{[27-28]}$.

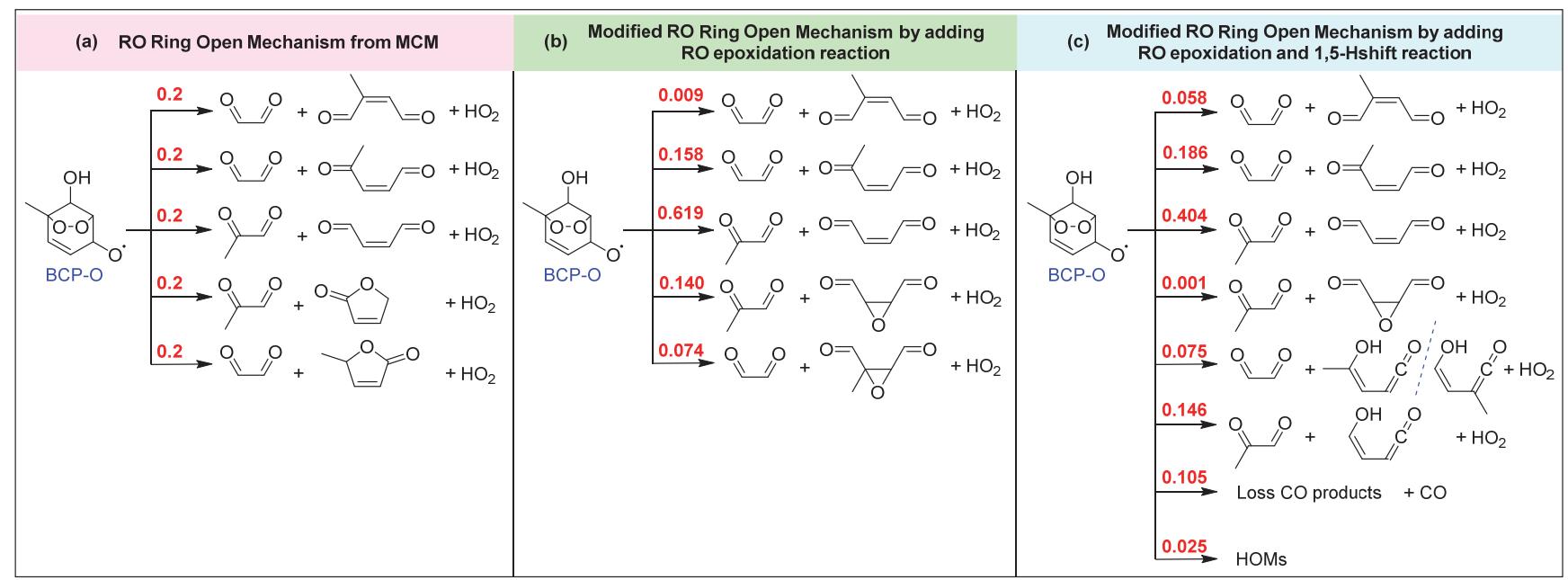

图式 9 芳香烃氧化过程 RO 的开环反应. 机制(a)参考 MCM (http://mcm.leeds.ac.uk/MCM-devel/roots.htt), 机制(b)参考 Wu 等 ${ }^{[62]}$ 的研究，机制(c) 参考 Wang 等 ${ }^{[28]}$ 和 $\mathrm{Xu}$ 等 ${ }^{[27]}$ 的研究

Scheme 9 RO ring open reactions during aromatic hydrocarbon oxidation mechanism. Mechanism (a) was refer to MCM (http://mcm.leeds.ac.uk/MCM-devel/roots.htt). Mechanism (b) refers to the research of Wu et al. ${ }^{[62]}$. Mechanism (c) refers to the research of Wang et al. ${ }^{[28]}$ and Xu et al. ${ }^{[27]}$ 


\subsection{1 双环过氧自由基氢转移理论}

近年来, 苯、甲苯和二甲苯氧化的烟雾箱研究发现 低 $\mathrm{NO}_{x}$ 条件下 $\mathrm{SOA}$ 产率明显高于高 $\mathrm{NO}_{x}$ 下的产率, $\mathrm{Ng}$ 等 ${ }^{[13]}$ 提出可能是由于低 $\mathrm{NO}_{x}$ 下双环过氧自由基 (BCP-OO)与 $\mathrm{HO}_{2}$ 反应形成了高 $\mathrm{SOA}$ 产率的 $\mathrm{ROOH}$ 所 致. 但 Dawson 等 ${ }^{[122]}(2016)$ 和 $\mathrm{Xu}$ 等 ${ }^{[123]}(2015)$ 的研究发 现, 在低 $\mathrm{NO}_{x}$ 条件下, 即使将 $\mathrm{BCP}-\mathrm{OO}$ 与 $\mathrm{HO}_{2}$ 的双分子 反应纳入 SOA 模型后，仍会估低芳香烃 $20 \%$ ～50\%的 SOA 产率. 针对这一问题, Wang 等 ${ }^{[51]}(2017)$ 的最新研究 发现, 在大气条件特别是低 $\mathrm{NO}_{x}$ 条件下, 双环 $\mathrm{RO}_{2}$ 的单 分子异构化反应(分子内氢转移)可以与其双分子反应竞 争, 生成非芳香烃的环状高氧化态有机物(HOMs), 对 SOA 的生成有重要影响. 此外, 氢转移过程中会伴随着 $\mathrm{OH}$ 自由基, 是 $\mathrm{OH}$ 自由基重要来源之一. 在异成二烯的 氧化反应研究中已经证实此种 $\mathrm{OH}$ 再生机制会加速 $\mathrm{HO}_{x}$ 自由基的循环 ${ }^{[121]}$, 但在芳香烃氧化过程中对 $\mathrm{HO}_{x}$ 自由 基的影响仍然有待探索. 芳香烃氧化过程中双环 $\mathrm{RO}_{2}$ 氢 转移反应如图式 10 所示. 研究指出甲苯、乙苯和丙苯 氧化过程中 $\mathrm{RO}_{2}$ 分子内氢转移的等效反应速率常数, 分 别为 $5.1 \times 10^{-6} \sim 2.6 \times 10^{-2} \mathrm{~s}^{-1}, 2.5 \times 10^{-3} \sim 7 \mathrm{~s}^{-1}$ 和 $8.8 \sim$ $14 \mathrm{~s}^{-1}$, 且芳香烃烷基取代基的点位差异对反应速率常 数的影响显著. 对于多取代基芳香烃, 由于具有较高的 BCP-OO 产率, 以及相对多的弱碳氢键 ${ }^{[26]}$, 它们发生 $\mathrm{H}$ 转移即自氧化反应(auto-oxidation)的几率会更大. 由此 可见 $\mathrm{BCP}-\mathrm{OO}$ 的氢转移反应途径在芳香烃低 $\mathrm{NO}_{x}$ 氧化 机理中不可忽视, 但是该途径存在较大争议, 至今并没 有纳入 MCM 机理.

\subsection{2 醛类化合物氢转移理论}

双环烷氧自由基(BCP-O)的主要后续反应为环氧闭 环反应和环断裂反应, 分别如图式 7 和图式 9 所示. 但 是 Wang 等 ${ }^{[28]}$ 理论计算结果表明仅有不足 $2 \%$ 的 BCP-O 自由基发生了环氧闭环反应. 此外, $\mathrm{Xu}$ 等 ${ }^{[27]}$ 通过烟雾箱 实验研究也发现环氧闭环反应产物的产率小于 $0.2 \%$, 也认为 $\mathrm{BCP}-\mathrm{O}$ 的环断裂反应是其后续的主导反应.

BCP-O 自由基发生环断裂反应会产生开环的烷氧 自由基(如图式 11 中的 $\mathrm{C}_{7} \mathrm{H}_{9} \mathrm{O}_{4} \cdot$ ). 经典机理主要认为烷 氧自由基发生环断裂反应只形成小分子醛类化合 物 ${ }^{[24-25,88,118]}$. 然而, 现有机理仍存在碳缺失的问题, 比 如模型模拟中显著低估了甲醛的浓度，低估程度达到 4 倍以上 ${ }^{[124-125]}$. Wang 等 ${ }^{[28]}$ (2020 年)最新的研究发现, 苯 氧化产生的大部分 $\mathrm{C}_{6} \mathrm{H}_{7} \mathrm{O}_{4} \cdot$ 烷氧自由基发生裂解时除直 接产生小分子二醛之外, 还能通过 1,5 -氢转移形成 $\mathrm{C}_{6} \mathrm{H}_{7} \mathrm{O}_{4}$-yl 烷基自由基，随后分解为中间的乙烯酮-烯醇 化合物，同时补充乙烯酮-烯醇与 $\mathrm{O}_{3}$ 和 $\mathrm{OH}$ 反应形成 $\mathrm{HCOOH}$ 的新途径, 有效解释了现有苯系物氧化机理中 甲酸二次源缺失的现象.

同年, Xu 等 ${ }^{[27]}(2020)$ 在研究苯的氧化机理时, 测试 了 $\mathrm{C}_{6} \mathrm{H}_{7} \mathrm{O}_{4}$ ・烷氧自由基的 1,5-氢转移过程. 研究发现, 虽 然新的 1,5-氢转移理论可以提高对芳香烃氧化的理解, 但 $\mathrm{C}_{6} \mathrm{H}_{7} \mathrm{O}_{4} \cdot$ 烷氧自由基的后续通道中仍然存在约 $20 \%$ 的 碳缺失情况. $\mathrm{Xu}$ 等发现除了 Wang 等提出的 $\mathrm{C}_{6} \mathrm{H}_{7} \mathrm{O}_{4}$-yl 烷基自由基可以分解成乙烯酮-烯醇化合物外，还有 $30 \%$ 的 $\mathrm{C}_{6} \mathrm{H}_{7} \mathrm{O}_{4}-\mathrm{yl}$ 会经历 CO-loss 生成低碳产物, $7 \%$ 的 $\mathrm{C}_{6} \mathrm{H}_{7} \mathrm{O}_{4}-\mathrm{yl}$ 会和 $\mathrm{O}_{2}$ 反应生成 HOMs. 但这一途径 2020 年

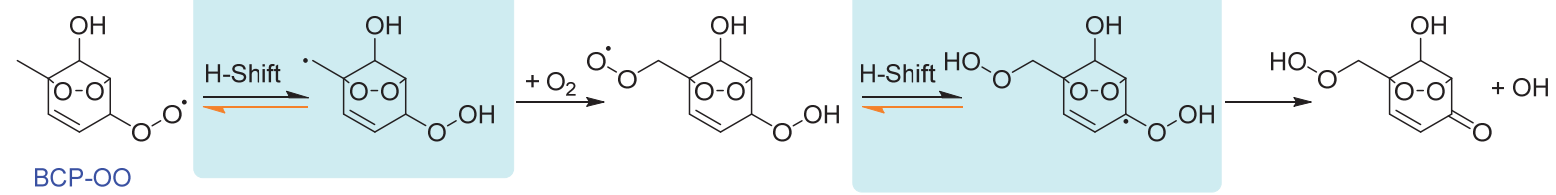

图式 10 芳香烃氧化过程中 $\mathrm{RO}_{2}$ 的 $\mathrm{H}$ 转移反应

Scheme $10 \quad \mathrm{RO}_{2} \mathrm{H}$-Migrations reaction during aromatic hydrocarbon oxidation mechanism

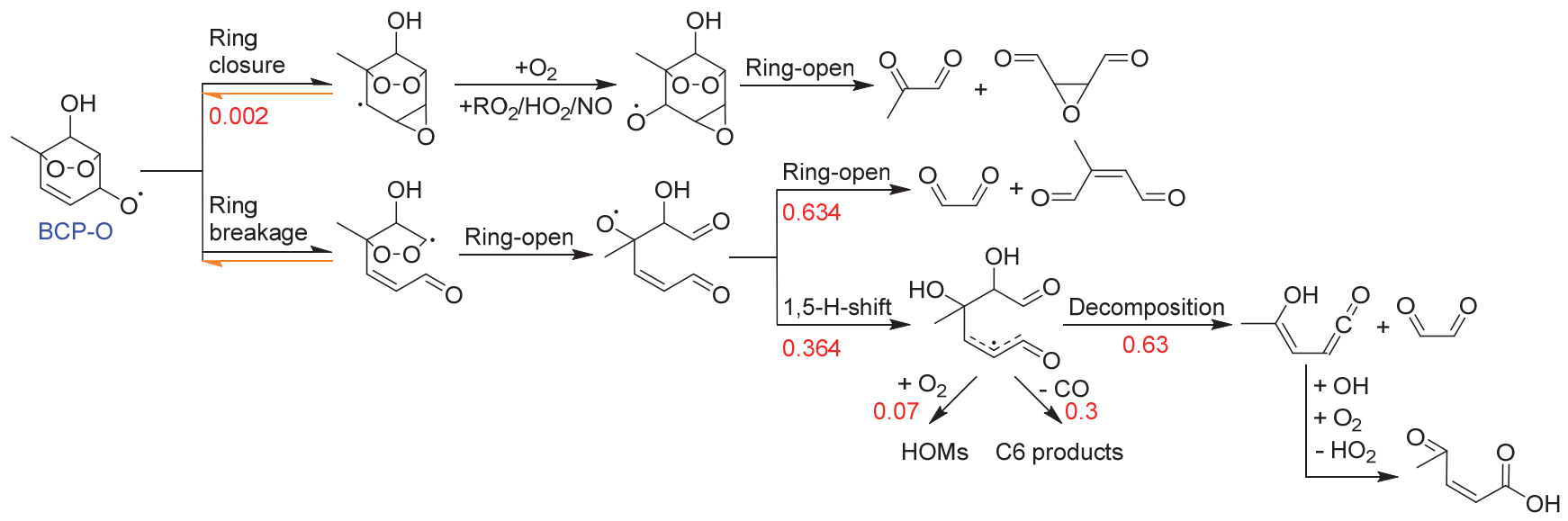

图式 11 芳香烃氧化过程中醛类化合物 $\mathrm{H}$ 转移反应 ${ }^{[27]}$

Scheme 11 Aldehydic H-shift reactions during aromatic hydrocarbon oxidation mechanism ${ }^{[27]}$ 
才于苯的氧化研究中提出, 各个步骤均具有不确定性, 值得进一步研究来验证. 根据苯氧化中的 1,5 -氢转移理 论推导出甲苯氧化过程中醛类化合物氢转移反应如图 式 11 所示.

\section{8 影响芳香烃大气氧化反应主要因素}

芳香烃氧化过程中各分支反应受多种因素的影响. 首先, 温度是影响芳香烃大气氧化初始反应的关键因 素，但在常温常压下, $\mathrm{OH}$ 自由基与芳香烃的加成途径 占主导地位 ${ }^{[4,62,66]}$. 而多项研究发现芳香烃 $\mathrm{OH}$ 加成产 物的后续反应主要受 $\mathrm{O}_{2}$ 浓度, $\mathrm{OH}$ 浓度和 $\mathrm{NO}_{x}$ 浓度的影 响 [27,34,60,126-127]. 在实际大气条件下, $\mathrm{NO}_{x}$ 浓度是影响芳 香烃氧化过程的主导因素. 低 $\mathrm{NO}_{x}$ 条件下 $\left(\mathrm{NO}_{x}<1\right.$ $\left.\mu \mathrm{g} \cdot \mathrm{m}^{-3}\right)$, 芳香烃氧化产物 $\mathrm{RO}_{2}$ 自由基可以分子内氢转 移生成 $\mathrm{HOMs}$, 并同时再生 $\mathrm{OH}$ 自由基, 加速 $\mathrm{HO}_{x}$ 自由 基的循环, 进而影响 $\mathrm{NO}-\mathrm{NO}_{2}-\mathrm{O}_{3}$ 的循环过程 ${ }^{[26]}$. 高 $\mathrm{NO}_{x}$ 条件下, 芳香烃氧化产生的 $\mathrm{RO}_{2}$ 可以被 $\mathrm{NO}$ 还原为 $\mathrm{RO}$ 和有机硝酸酯 $\left(\mathrm{RONO}_{2}\right)$, 其中, $\mathrm{RO}$ 可以进行环断裂反

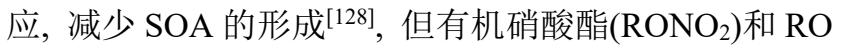
环断裂形成的环氧化物和二羰基醛等物质, 很容易分配 到气溶胶相并促进 SOA 的生长 ${ }^{[129]}$. $\mathrm{Ng}$ 等 ${ }^{[13]}$ (2007)通过 对苯、甲苯和二甲苯氧化的烟雾箱研究发现, 低 $\mathrm{NO}_{x}$ 条 件下苯、甲苯和二甲苯的 SOA 产率(分别为 $37 \%$ 、 $30 \%$ 和 $36 \%$ ) 远高于高 $\mathrm{NO}_{x}$ 条件下的 $\mathrm{SOA}$ 产率(分别为 $28 \%$ 、 $11 \%$ 和 $6 \%$ ). $\mathrm{NO}_{x}$ 浓度还会影响芳香烃氧化过程中 HOMs 的产率和组成. Garmash 等 ${ }^{[127]}(2020)$ 通过对苯氧化的流 动管研究发现, 在无 $\mathrm{NO}_{x}$ 参与的条件下, $1 \mathrm{~mol}$ 苯的 HOMs 产率在 $4.1 \% \sim 14.0 \%$ 之间, 显著高于 $\mathrm{NO}_{x}$ 参与的 情况, 且苯的 HOMs 产率对 $\mathrm{OH}$ 浓度有很强的依赖性. 此外, $\mathrm{NO}_{x}$ 浓度对芳香烃氧化过程中新粒子的形成也有 着重要影响, Qi 等 ${ }^{[130]}(2020)$ 进行了不同 $\mathrm{NO}_{x}$ 条件下的甲 苯氧化实验发现, 在低 $\mathrm{NO}_{x}$ 条件下 $\left(\mathrm{NO}_{x}\right.$ /甲苯 $\left.<0.16\right)$, $\mathrm{NO}_{x}$ 促进了新粒子的形成, 随着 $\mathrm{NO}_{x}$ 的升高, 甲苯的 $\mathrm{SOA}$ 产率也随之升高; 而在高 $\mathrm{NO}_{x}$ 条件下 $\left(\mathrm{NO}_{x}\right.$ /甲苯 $>$ $0.16), \mathrm{NO}_{x}$ 则抑制了新粒子的形成, 随着 $\mathrm{NO}_{x}$ 的升高, 甲苯的 SOA 产率也随之降低.

\section{3 芳香烃氧化机理的研究手段}

芳香烃氧化机理的研究手段可概括为基于量子化 学理论计算的研究和基于实验室模拟的研究, 实验室研 究又可以分为基于氧化流动反应器(oxidation flow reactor, OFR)的研究和基于环境烟雾箱的研究. 图 4 给出了 在三种研究手段的帮助下, 近 20 年内芳香烃氧化机理 的研究进程.

\section{1 基于理论计算的芳香烃氧化机理研究}

在芳香烃氧化机理研究领域内, 理论计算发挥了十 分重要的作用(图 4). 芳香烃氧化过程中存在大量的过
氧自由基，这些过氧自由基的反应活性相对较高，浓度 相对较低，在检测方面具有极大的挑战. 在芳香烃氧化 的实验研究中，仅有很少量的活性自由基中间体能够被 检测，比如 Birdsall 等 ${ }^{[34,60]}$ 采用化学电离质谱装置首次 检测到甲苯氧化过程中双环过氧自由基中间体的存在， 同时证实了早期理论预测的重要性. 大多数的过氧自由 基产物由于其高反应性、不稳定性或光降解性，使得其 稳态浓度极低而无法被检测出来，即使检测出来不同实 验测得的结果也相差较大. 此外, 对于绝大多数过氧自 由基的后续反应来说，反应过程中会产生过渡态结构的 产物, 目前很难用实验手段检测. 为了弥补实验检测条 件的不足, 科学家常常借助量子化学理论计算对芳香烃 的氧化降解机理进行研究, 试图揭示氧化降解机理, 预 测未检测产物结构.

理论计算在芳香烃氧化机理研究中的主要贡献在 于借助量子化学计算和化学反应动力学研究给出各类 芳香烃详细微观的反应机制及反应速率(图 4). 在苯的 氧化机理研究中, Glowacki 等 ${ }^{[131]}$ 基于微正则过渡态理 论 RRKM-ME 方法计算, 得出苯的 $\mathrm{OH}$ 加成产物可以与 $\mathrm{O}_{2}$ 发生摘氢反应形成苯酚. Wang 等 ${ }^{[24]}$ 基于 BH\&HLYP/6-311++G(2df,2p) 的理论计算水平, 得出 了苯氧化过程中烷基自由基除发生环断裂反应，还可以 发生环闭合反应，从理论上解释了实验中检测的乙二醛 产率高于丁烯二醛的现象. Wang 等 ${ }^{[28]}$ 基于 DFT-M06$2 \mathrm{X} / 6-311++\mathrm{G}(2 \mathrm{df}, 2 \mathrm{p})$ 的理论计算水平, 修正了苯氧化 过程中双环烷氧基环闭合反应的重要性，指出 BCP-O 的环断裂反应为后续的主导反应，并提出环断裂产物后 续醛类 1,5-氢转移新途径. 在甲苯的氧化机理研究中, Suh 等[69]基于 B3LYP/6-31G(d,p)的理论计算水平, 预测 $\mathrm{OH}$ 更有利于加成在甲苯的邻位和对位, 而 $\mathrm{OH}$ 加成产 物与 $\mathrm{O}_{2}$ 反应形成的过氧自由基会迅速环化形成双环烷 基自由基 ${ }^{[73]}$. $\mathrm{Wu}$ 等 ${ }^{[62]}$ 基于 DFT-M06-2X/6-311 ++ $\mathrm{G}(2 \mathrm{df}, 2 \mathrm{p})$ 的理论计算水平, 给出较完整的甲苯氧化机理 框架, 并指出双环烷氧基环闭合反应在甲苯氧化过程中 的重要性. 在二甲苯的氧化机理研究中, Fan 和 Zhang ${ }^{[70]}$ 采用密度泛函理论(DFT)计算方法, 得出 $\mathrm{OH}$ 倾向于加 成间二甲苯的邻位; Huang 等[132]使用 DFT 和经典过渡 态速率理论(CTST)计算方法, 得出 $\mathrm{OH}$ 倾向于加成间邻 二甲苯的邻位, 加成产物会与 $\mathrm{O}_{2}$ 发生加成反应，随后分 子内重排成双环烷基自由基，后续会与 $\mathrm{O}_{2}$ 结合形成双 环过氧自由基或开环形成环氧桥烷氧自由基. 在三甲苯 的氧化机理研究中, Li 和 Wang ${ }^{[133]}$ 基于 M06-2X//6-311 $++\mathrm{G}(2 \mathrm{df}, 2 \mathrm{p})$ 的理论计算水平, 给出较完整的 1,2,4-三 甲苯氧化机理框架, Ponnusamy 等 ${ }^{[134]}$ 基于 M06-2X, 26 $\mathrm{w}-\mathrm{B} 97 \mathrm{XD} 27$ 和 MPW1K28 在 6-311+ $+\mathrm{G}(\mathrm{d}, \mathrm{p})$ 的理论计 算水平, 给出较完整的 $1,3,5$-三甲苯氧化机理框架. 


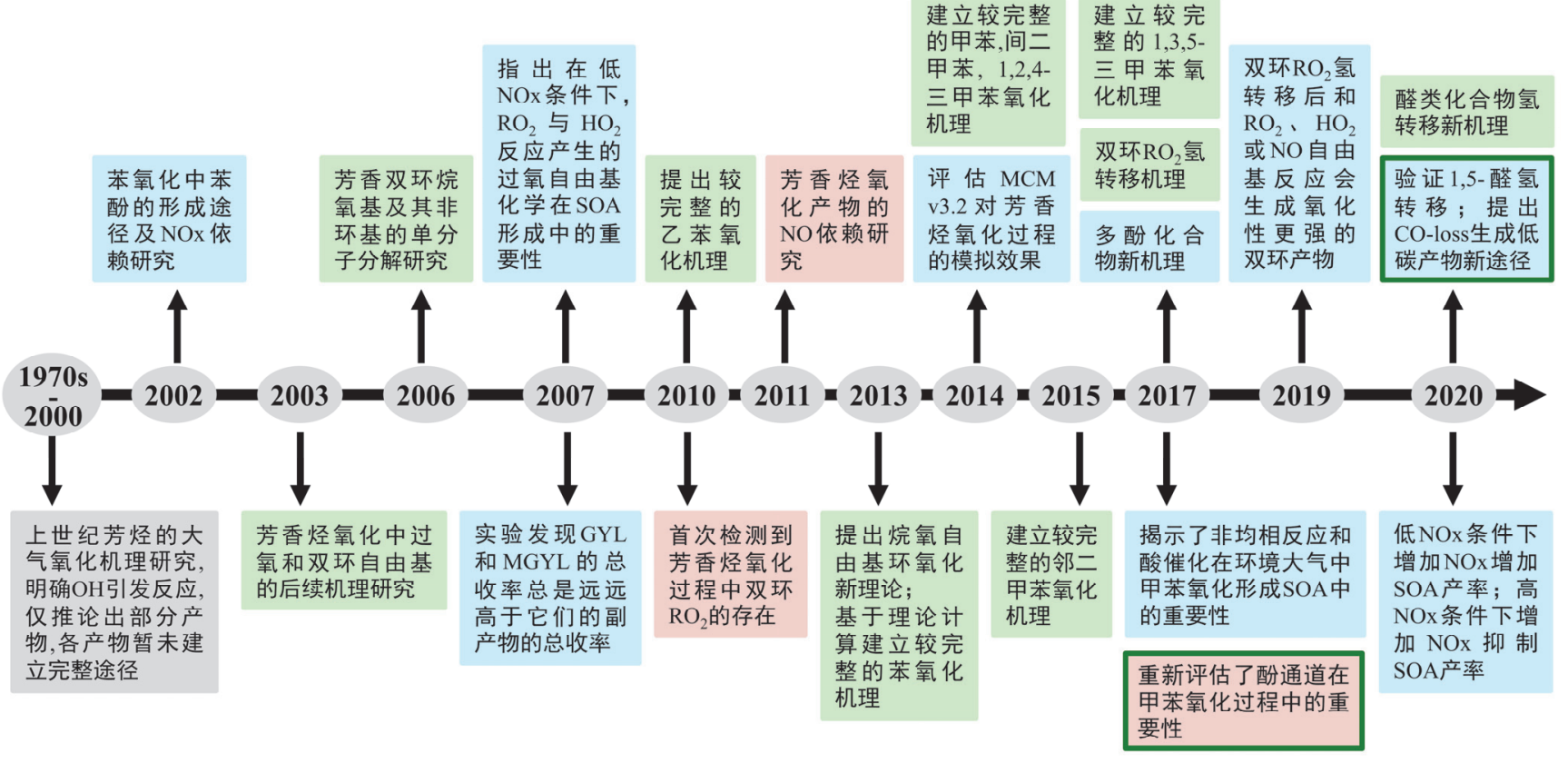

图 4 近 20 年芳香烃氧化机理研究发展历程图 ${ }^{[13,24,26-29,34,37-38,51,60,62,73,82,118,130,133-138]}$. 图中的年份代表了参考文献的发布年份

Figure 4 Development of researches on oxidation mechanism of aromatic hydrocarbons in recent 20 years ${ }^{[13,24,26-29,34,37-38,51,60,62,73,82,118,130,133-138]}$. The year in the figure represents the references' publish year

综上，近年来基于理论计算的研究，已经获得苯、 甲苯、二甲苯和三甲苯等芳香烃相对完整的机理构架. 但是由于理论计算的方法和计算水平参差不齐, 预测机 理的不确定性较大, 各种预测新理论均需要实验室的进 一步验证.

\section{2 基于实验室的芳香烃氧化机理研究}

在基于实验室的芳香烃氧化研究中, 环境烟雾箱具 有能够排除复杂的气象、地形等影响因素, 从而在可以 控制的条件下模拟大气中的化学过程, 获得反应的机制 机理的优点 ${ }^{[139-142]}$. 从近年来国内外基于烟雾箱的芳香 烃氧化机理研究中可以看出 ${ }^{[13,25,27,29,37,58,130,137,138,143]}$, 通 常烟雾箱采用特氟龙等吸附性较差的膜材料构成密闭 反应腔体, 通过向箱内通入定量的前体物(例如芳香烃 和 $\mathrm{NO}_{x}$ ) 以及 $\mathrm{OH}$ 自由基源(例如气态亚硝酸或 $\mathrm{H}_{2} \mathrm{O}_{2}$ ), 利 用太阳光源或黑灯模拟太阳辐射, 来模拟芳香烃在实际 大气中的气相氧化降解过程. Bloss 等 ${ }^{[58]}$ 在 EUPHORE 烟 雾箱中进行了苯、甲苯、对二甲苯和 $1,3,5$-三甲苯的氧 化实验, 讨论了 $\mathrm{MCM} 3$ 和 $\mathrm{MCM} 3.1$ 机理对实测数据的 模拟情况, 发现 MCM3 和 MCM3.1 模型结果存在高估 $\mathrm{O}_{3}$ 浓度和低估 $\mathrm{OH}$ 模拟值的现象. Nehr 等 ${ }^{[137]}$ 在 SAPHIR 烟雾箱中进行了苯、甲苯、对二甲苯和 1,2,4-三甲苯的 氧化实验, 通过比较观测结果和 MCM3.2 的模拟结果, 发现 MCM3.2 模型低估了芳香烃氧化研究中的 $\mathrm{OH}$ 自由 基浓度, 高估了二次产物的 $\mathrm{OH}$ 反应活性, 低估了过氧 自由基浓度. 为了研究芳香烃氧化降解生成 SOA 的过
程, 部分研究向烟雾箱体系内通入了硫酸铵等种子气溶

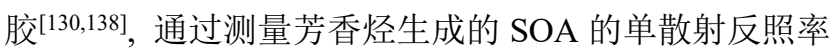
等气溶胶光学特性来全面评估芳香烃氧化生成 SOA 的 环境效应 ${ }^{[130]}$.

相较于烟雾箱, 氧化流动管反应器具有体积小、反 应时间快的特点. 氧化流动管采用派热克斯玻璃、特氟 龙或石英等材质, 通过 $\mathrm{OH}$ 发生器产生比实际大气高出 几个数量级的 $\mathrm{OH}$ 自由基, 从而实现在几十秒钟至几分 钟的时间内模拟实际大气中芳香烃几天至几周的变 化 ${ }^{[34,38,60,144]}$. 其研究重点在于通过测量经由氧化流动管 反应器后气体中的芳香烃浓度和 SOA 的理化性质, 进 而研究芳香烃氧化过程中 SOA 的形成和老化过 程 ${ }^{[144-145]}$.

近年来, 基于实验室的芳香烃氧化机理研究在现有 机理模拟效果评估和环境效应研究方面发挥着重要的 作用. 但基于实验室的研究常常依托于现有氧化理论框 架的指引和中间氧化产物的准确定性定量. 因此, 将理 论计算和实验室研究相互结合, 是未来研究芳香烃氧化 过程及其环境效应的新方向.

\section{4 芳香烃氧化主要中间产物及其测量方法}

芳香烃氧化可以产生多种中间产物，根据氧化途径 可以将氧化中间产物分为以下五类, 分别为: (1)醛通道: 苯甲醛、甲基苯甲醛等芳香醛类化合物，(2)酚通道: 苯 酚、甲酚、多羟基芳香酚类和硝基苯酚类化合物，(3)双 
环过氧自由基通道: 甲醛、乙二醛、甲基乙二醛、有机 硝酸酯、酰基过氧硝酸盐, (4)环氧化物通道: 环氧化物, (5)自由基类: 包括 $\mathrm{OH}$ 自由基, $\mathrm{HO}_{2}$ 自由基和 $\mathrm{RO}_{2}$ 自由 基. 根据芳香烃氧化产物的性质可以将氧化产物分为以 下六类: (1)二羰基化合物: 包括乙二醛、甲基乙二醛等, (2)多差基类有机物: 包括甲酚、苯酚、二羟基甲苯、三 羟基甲苯和四羟基甲苯等，(3)酸类、多官能团化合物: 包括苯甲酸、一羟基-甲基苯醌等, (4)高氧化态化合物 (highly oxygenated organic molecules, HOMs): HOM 是 双环过氧自由基通过分子内氢转移从而发生自氧化反 应形成的含有 6 个或者超过 6 个氧原子的低挥发性有机 物，(5)含氮含氧化合物：包括硝基苯酚类化合物，(6)自 由基类: 包括 $\mathrm{OH}$ 自由基, $\mathrm{HO}_{2}$ 自由基和 $\mathrm{RO}_{2}$ 自由基. 对 近 20 年芳香烃氧化中间产物的测量技术进行归纳总结, 如图 5 所示. 质谱法和光谱法是芳香烃氧化中间产物最 常用的测量技术. 其中质子转移反应质谱法 (proton-transfer-reaction mass spectrometry, PTR-MS)主 要可以用于二羰基化合物、酚类、苯甲醛类、多羟基化 合物、含氮含氧化合物等分子量相对较小的挥发性产物. 化学电离质谱(CIMS)技术可以覆盖几乎所有的中间产 物的测量范围, 包括半挥发性和低挥发性、分子量相对 较高的产物, 但需要针对不同类型的中间产物选择合适 的离子源. 由于单一离子源的质谱手段并不能比较全面 的覆盖芳香烃氧化中间产物的测量, 且 PTR-MS 和 CIMS 虽然可以同步测量大量物种, 但是无法区分同分 异构体. 因此越来越多的研究将多种离子源的质谱进行
联用或者与色谱技术联用，在芳香烃氧化机理研究中发 挥了重要的作用. 光谱技术主要应用在 $\mathrm{HO}_{x}$ 自由基、总 $\mathrm{RO}_{2}$ 自由基、二羰基化合物以及总过氧化物硝酸盐和总 烷基硝酸盐的测量上 ${ }^{[146]}$. 光谱技术虽然在测量自由基 和小分子二羰基物上具有一定优势，但是由于其配置光 源的特定性，很难同时测量多种中间氧化产物，需要与 质谱仪相互配合使用. 因此多种测量技术的联用也成为 了未来芳香烃氧化中间产物测量领域的发展方向.

通过对现阶段多种芳香烃氧化主要途径分支比研 究发现(图 6a), 芳香烃氧化过程醛通道占比较小, 且各 研究结果呈现较好的一致性; 酚途径和双环 $\mathrm{RO}_{2}$ 途径分 支比占比较大, 且各研究中存在较大争议; 而环氧化物 通道虽然占比较小, 但认识局限于理论计算研究, 实验 室研究中很少可以观测到环氧化物的产生, 因此争议较 大. 值得注意的是，随着取代基的增多，芳香烃氧化过 程呈现酚途径占比逐渐减少, 双环 $\mathrm{RO}_{2}$ 途径逐渐增加的 特点. 通过对比各研究中芳香烃氧化产物的产率发现, 大部分实验室研究中测出的中间产物有限, 少数研究能 够同时测量出多酚、苯甲醛、乙二醛/甲基乙二醛低聚物、 有机硝酸酯、硝基苯酚类化合物、酰基过氧硝酸盐、环 氧化物等重要的氧化产物, 且不同研究中由于实验条件 (特别是 $\mathrm{NO}_{x}$ 浓度)的不同，导致芳香烃氧化中间产物的 产率均存在较大的差异(图 6b). 这成为了芳香烃氧化机 理研究过程中的一大重要阻碍. 因此, 实现多种测量技 术的高效联用，完善对氧化中间产物的精准测量，对芳 香烃氧化机理的研究至关重要.

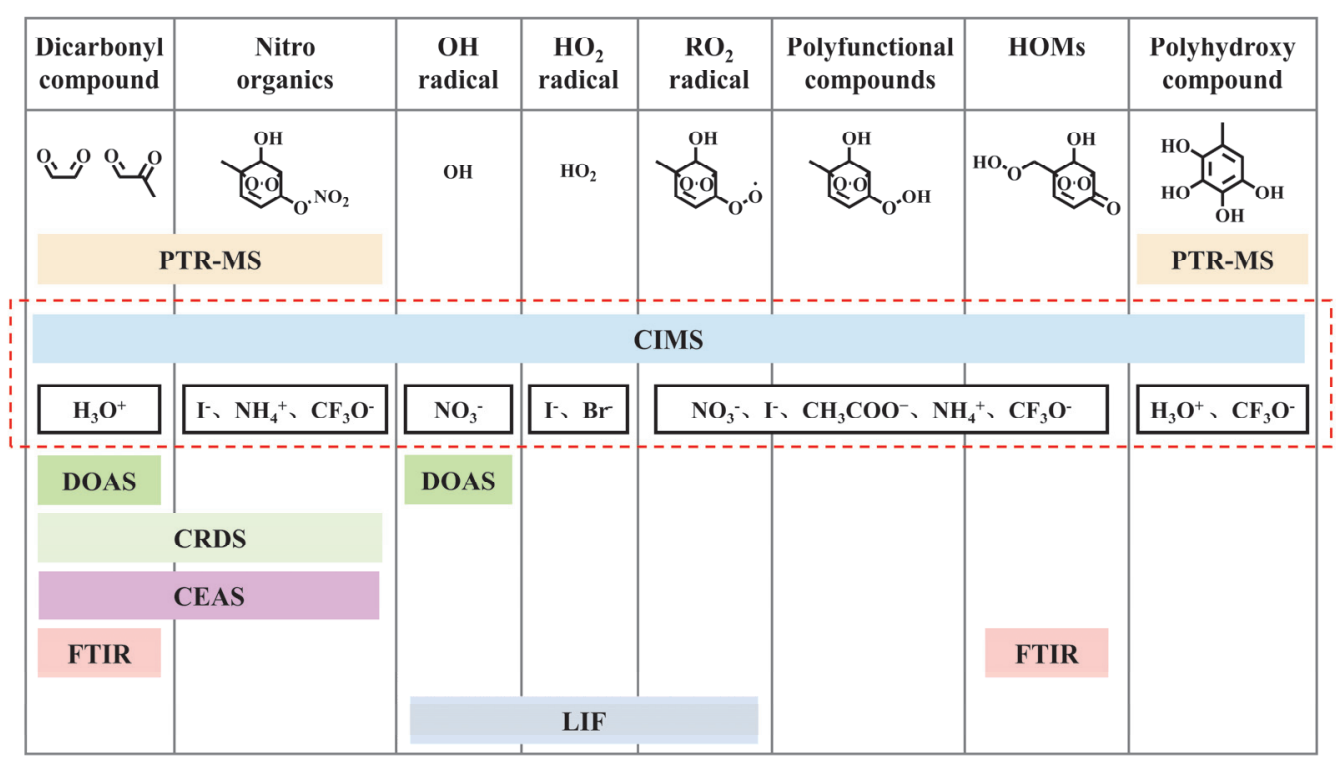

图 5 芳香烃氧化主要中间产物及自由基的常用测量方法 ${ }^{[26-27,29,45,147-168]}$. 图中的化合物分子式仅为每类中间氧化产物的代表. PTR 代表质子转移 反应质谱, CIMS 代表化学电离质谱, DOAS 代表差分光学吸收光谱, CRDS 代表腔衰荡光谱, CEAS 代表腔增强吸收光谱, FTIR 代表傅里叶变换红 外光谱, LIF 代表激光诱导苂光技术

Figure 5 Common measure methods of the main aromatic hydrocarbon oxidation intermediate product and radicals ${ }^{[26-27,29,45,147-168]}$. The compounds in the figure are examples of each type of intermediate oxidation product. PTR: proton-transfer-reaction mass spectrometry, CIMS: chemical ionization mass spectrometer, DOAS: differential optical absorption spectroscopy, CRDS: cavity ring down spectroscopy, CEAS: cavity enhanced absorption spectroscopy, FTIR: Fourier transform infrared, LIF: laser-induced fluorescence 


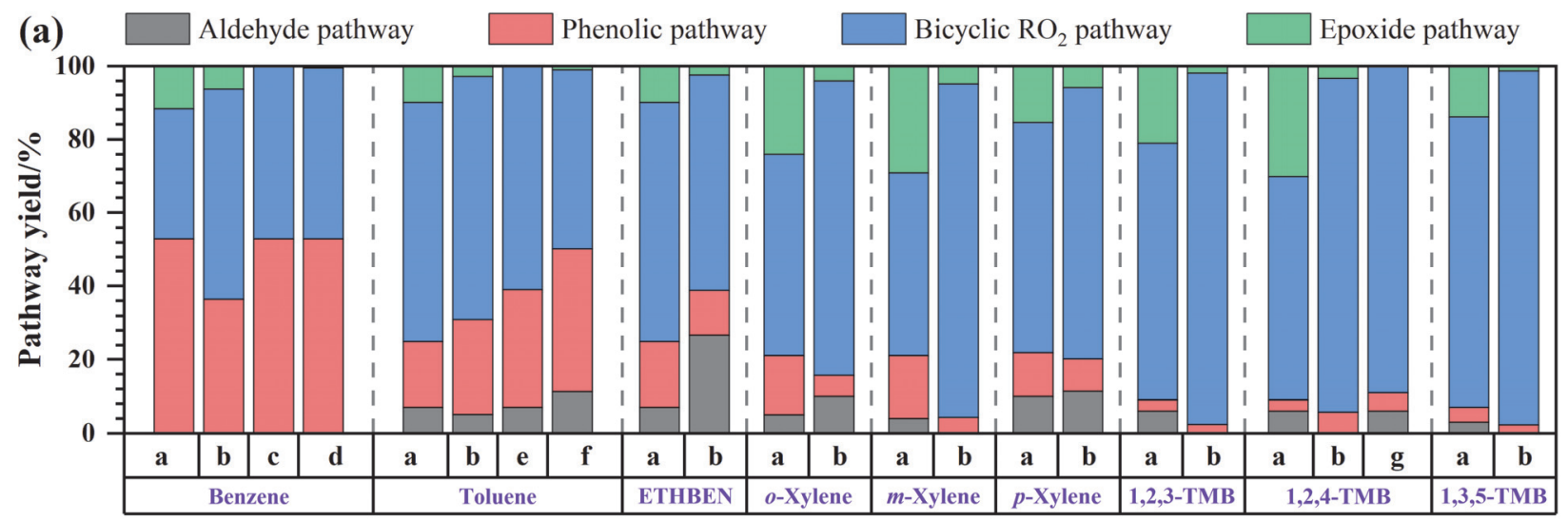

(b) $\square 25 \% \sim 75 \% \quad$ I Min Max - Median Line $\square$ Mean $\quad$ Literature values

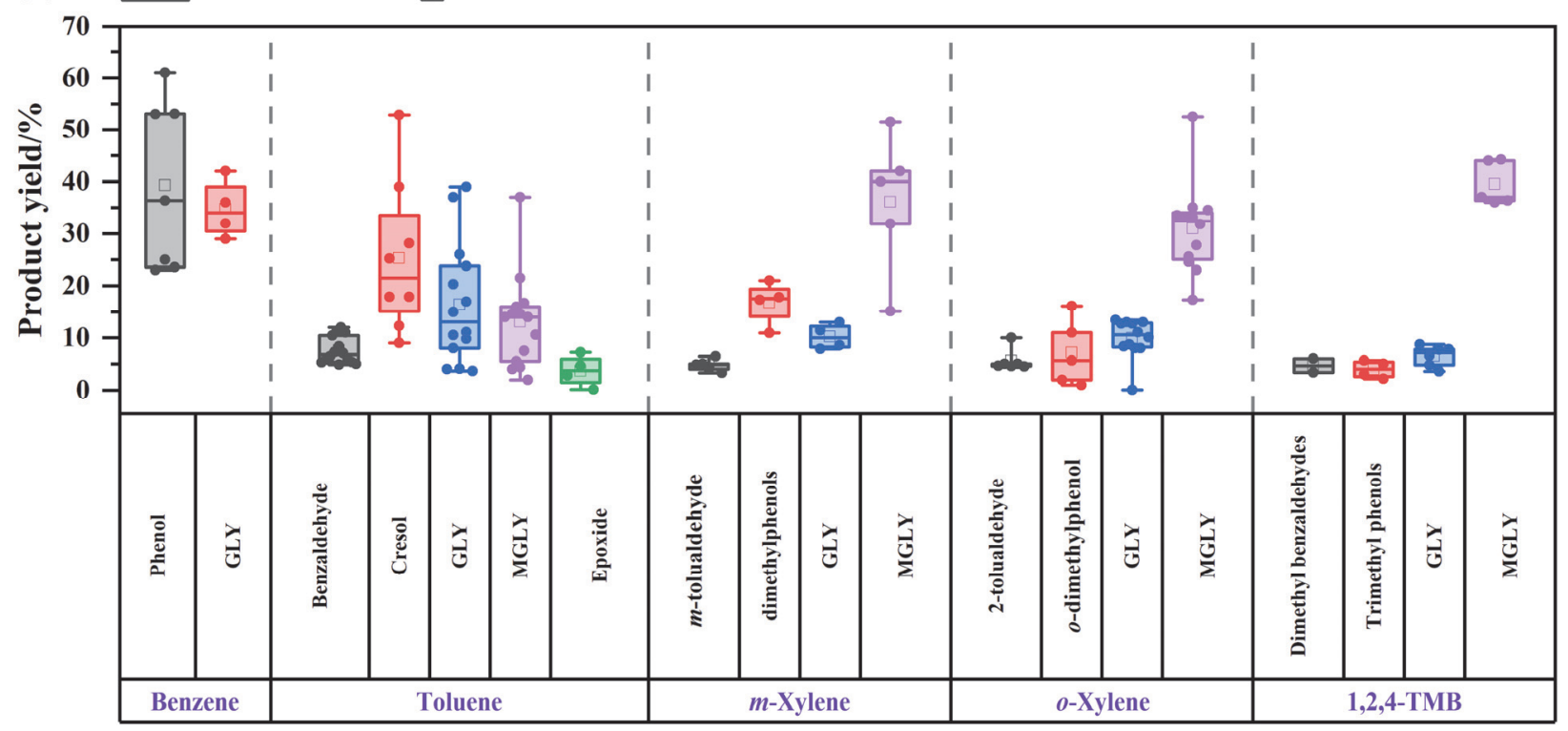

图 6 理论计算和实验室研究中芳香烃氧化主要氧化途径占比及中间产物产率汇总 $[24-25,27,30-41,44-48,50,62,119,133,135]$. ETHBEN 代表乙苯, a 代表 MCM 机 制的结果(http://mcm.york.ac.uk/), b 代表 Birdsall 和 Elrod 等 ${ }^{[34}$ 的研究结果, $\mathrm{c}$ 代表 Wang 等 ${ }^{[2]}$ 的研究结果, $\mathrm{d}$ 代表 $\mathrm{Xu}$ 等 ${ }^{[27]}$ 的研究结果, e 代表 Wu 等 ${ }^{[2]}$ 的研究结果, $\mathrm{f}$ 代表 $\mathrm{Ji}$ 等 ${ }^{[38]}$ 的研究结果, $\mathrm{g}$ 代表 $\mathrm{Li}$ 和 Wang 等 ${ }^{[133]}$ 的研究结果

Figure 6 Summary of the proportion of the main oxidation pathways of aromatic hydrocarbons and the yield of intermediate products in theoretical calculation and laboratory research ${ }^{[24-25,27,30-41,44-48,50,62,119,133,135]}$. ETHBEN: ethyl benzene, a represent the result of MCM mechanism (http://mcm.york.ac.uk/), $\mathrm{b}$ represent the study of Birdsall and Elrod ${ }^{[34]}$, c represent the study of Wang et al. ${ }^{[24]}, \mathrm{d}$ represent the study of Xu et al. ${ }^{[27]}$, e represent the study of Wu et $a l . .^{[62]}$, frepresent the study of Ji et al. ${ }^{[38]}$, g represent the study of Li and Wang ${ }^{[133]}$

\section{5 总结与展望}

芳香烃作为主导城市大气臭氧和二次有机气溶胶 生成的重要前体物, 对大气污染、气候变化和人类健康 具有重要影响. 现阶段对芳香烃的氧化机理研究只能构 建其大致理论框架, 很多影响臭氧和二次有机气溶胶生 成的关键反应并未得到实验室研究的一致认可, 且不同 研究或不同 $\mathrm{NO}_{x}$ 条件下常见的中间氧化产物的产率均 存在较大的差异. 因此芳香烃氧化机理及其环境效应的 研究已经成为当前大气环境化学领域最具挑战的热点 研究之一.

本综述立足于芳香烃在大气中的氧化反应过程, 总 结了近年来芳香烃氧化机理的研究成果, 重点关注芳香
烃氧化反应研究中的新发现和新理论. 研究发现大气中 芳香族化合物氧化的起始是由 $\mathrm{OH}$ 自由基的反应主导 的, 反应主要分为摘氢和加成两种途径. 摘氢途径占比 较小，是芳香醛类化合物的主要生成途径. 加成途径占 比较大, 产物 $\mathrm{C}-\mathrm{yl}$ 烷基自由基又可以和 $\mathrm{O}_{2}$ 发生摘氢和 加成两种反应. 当 $\mathrm{C}-\mathrm{yl}$ 烷基自由基与 $\mathrm{O}_{2}$ 发生摘氢反应 会形成酚类化合物. 当 $\mathrm{C}-\mathrm{yl}$ 烷基自由基与 $\mathrm{O}_{2}$ 发生多次 加成反应时, 会产生大量的 $\mathrm{RO}_{2}$ 自由基, 这些 $\mathrm{RO}_{2}$ 自由 基可以与 $\mathrm{NO}, \mathrm{HO}_{2}$ 和 $\mathrm{RO}_{2}$ 自由基发生双分子反应形成 $\mathrm{RO}$ 自由基、有机硝酸酯、过氧化物、醌类化合物等，部 分双环 $\mathrm{RO}_{2}$ 还可以进行分子内氢转移形成更高氧化态 低挥发性的 HOMs. 双分子反应产生的 RO 自由基的命 运主要是由烷氧基环氧化和环断裂之间的竞争决定的. 
而 RO 环断裂产生的烷氧自由基后续不仅可以分解形成 小分子醛类化合物, 可以通过 1,5 醛类氢转移反应和 CO-loss 反应生成烯酮-烯醇化合物和低碳化合物. 芳香 烃的氧化反应主要受温度, $\mathrm{O}_{2}$ 浓度, $\mathrm{OH}$ 浓度和 $\mathrm{NO}_{x}$ 浓度 的影响 $[27,34,60,126-127]$. 其中, 实际大气条件下, $\mathrm{NO}_{x}$ 浓度 是影响芳香烃氧化过程的主导因素. 此外, 本文还简要 总结了芳香烃大气氧化机理的研究方法和氧化中间产 物的测量技术. 研究发现基于理论计算的研究和基于实 验室的研究是芳香烃氧化机理的主要研究手段, 而质谱 法和光谱法是芳香烃氧化中间产物最常用的测量技术. 通过对芳香烃氧化机理研究的深入认识, 本文针对芳香 烃氧化理论的后续研究做出以下展望:

(1)进一步完善芳香烃的氧化机理：随着芳香烃氧 化机理研究不断发展, 出现了多种新机理, 其中比较有 代表性的分别是: 酚途径形成多羟基化合物、烷氧自由 基环氧化、双环过氧自由基分子内氢转移、醛类化合物 氢转移、CO-loss 生成低碳产物. 但现有机理研究之间存 在较大争议, 并不能完全解释芳香烃氧化过程中的 20\% 50\%的碳缺失和 30\% 50\%的自由基不闭合问题, 且不同 $\mathrm{NO}_{x}$ 条件下芳香烃氧化产物差异较大, 导致后续 臭氧与 SOA 形成机制不明, 亟需进一步研究完善.

(2)加强芳香烃氧化产物的精准测量: 现有实验研 究可以准确定量的中间产物有限, 极少有研究能够同时 测量出醛通道、酚通道、双环过氧自由基通道、环氧化 物通道的多代氧化产物及自由基，且存在约超过 $20 \%$ 的 芳香烃氧化中间产物未能被识别. 质谱法和光谱法虽然 是芳香烃氧化产物的主要测量手段, 但在同步测量和精 准定性定量上均存在不同技术弱点, 是亟需攻克的难 点. 此外, 多种测量技术的联用也成为了未来芳香烃氧 化中间产物测量领域的前沿发展方向.

(3)探究芳香烃氧化在实际大气中的环境效应: 实 际大气环境呈现生物源和人为源混合的复杂氧化体系, 不同前体物之间的氧化过程存在互相影响 ${ }^{[169]}$. 因此在 完善芳香烃氧化机理的前提下, 采用实际大气中重要前 体物比例进行复杂混合体系中芳香烃氧化效应分析, 参 数化其对臭氧和二次有机气溶胶的生成影响, 对改善空 气质量预测模型具有重要的意义.

\section{作者简介}

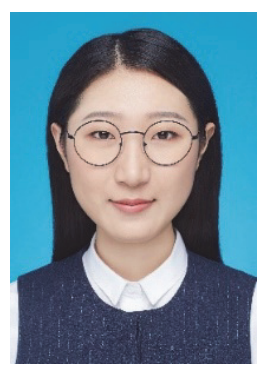

宋梦迪, 博士研究生, 2019 年进入北京大学环境科学与工 程学院. 主要研究方向为芳香烃氧化机理及环境效应, 大气
挥发性有机物的特征、来源与二次转化, 臭氧污染成因.

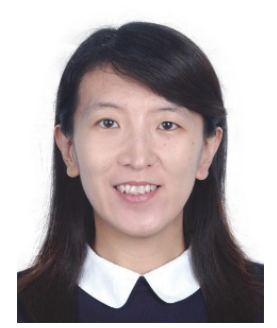

刘莹, 博士, 北京大学环境科学与工程学院副研究员. 2007 年获北京大学博士学位. 主要研究方向为城市和区域大 气有机碳的活性和来源、化学行为及环境影响等, 包括健全和 完善有机物在线质谱技术, 开展我国高污染和强氧化性条件 下含氧有机物的二次转化机制和化学演变特征、活性有机物 的来源及其对二次污染贡献、二次有机气溶胶形成机制等关 键科学问题的研究. 承担和参加多项国家自然科学基金、国家 重点研发计划、环保部公益项目等.

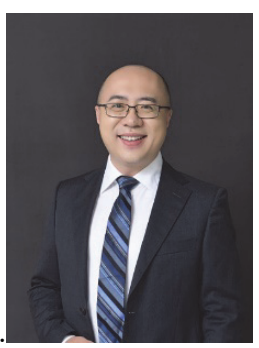

李歆, 博士, 北京大学环境科学与工程学院青年千人计 划研究员, 博士生导师. 2010 年获得北京大学博士学位, 2014 年9月２016 年6月就职于德国 Juelich 研究中心对流层研究 所. 2016 年获得北京大学职位. 主要研究方向为大气活性物 种的在线监测技术与来源转化机制, 致力于大气氧化性构成 和演变特征、一次污染物大气氧化产生二次污染的化学反应 机制、识别影响大气氧化进程的关键化学和物理要素等研究. 承担和参与多项国家自然科学基金委项目和国家重点研发计 划项目.

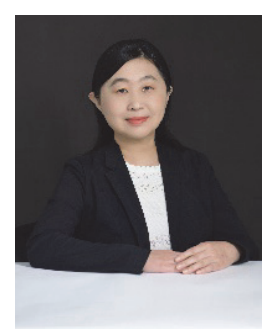

陆思华, 北京大学环境科学与工程学院, 教授级高工. 主 要研究方向为区域大气有机污染物的来源、转化、环境影响 等. 研究工作主要包括: 城市和区域大气 VOCs 的组成特征及 环境影响、VOCs 重点行业污染源排放特征及污染来源解析、 大气中 VOCs 和 SVOCs 分析测试技术及质控等. 近年来, 作 为课题负责人或课题骨千，负责和参加了大气重污染成因与 治理公关项目、科技部大气重点研发计划、国家环保公益性 行业科研专项及横向协作等多项科研工作.

\section{References}

[1] Guo, S.; Hu, M.; Zamora, M. L.; Peng, J.; Shang, D.; Zheng, J.; Du, Z.; Wu, Z.; Shao, M.; Zeng, L.; Molina, M. J.; Zhang, R. Proc. Natl. 
Acad. Sci. U. S. A. 2014, 111, 17373.

[2] Huang, R.-J.; Zhang, Y.; Bozzetti, C.; Ho, K.-F.; Cao, J.-J.; Han, Y.; Daellenbach, K. R.; Slowik, J. G.; Platt, S. M.; Canonaco, F.; Zotter, P.; Wolf, R.; Pieber, S. M.; Bruns, E. A.; Crippa, M.; Ciarelli, G.; Piazzalunga, A.; Schwikowski, M.; Abbaszade, G.; Schnelle-Kreis, J.; Zimmermann, R.; An, Z.; Szidat, S.; Baltensperger, U.; El Haddad, I.; Prevot, A. S. H. Nature 2014, 514, 218.

[3] Li, M.; Zhang, Q.; Zheng, B.; Tong, D.; Lei, Y.; Liu, F.; Hong, C. P.; Kang, S. C.; Yan, L.; Zhang, Y. X.; Bo, Y.; Su, H.; Cheng, Y. F.; He, K. B. Atmos. Chem. Phys. 2019, 19, 8897.

[4] Calvert, J. G.; Atkinson, R.; Becker, K. H.; Kamens, R. M.; Seinfeld, J. H.; Wallington, T. G.; Yarwood, G. The Mechanisms of Atmospheric Oxidation of Aromatic Hydrocarbons, Oxford University Press, New York, 2002.

[5] Liu, Y.; Shao, M.; Fu, L.; Lu, S.; Zeng, L.; Tang, D. Atmos. Environ. 2008, 42, 6247.

[6] Hu, D.; Tolocka, M.; Li, Q.; Kamens, R. M. Atmos. Environ. 2007, $41,6478$.

[7] Wu, R.; Xie, S. Environ. Sci. Technol. 2017, 51, 2574.

[8] Wu, F.; Wang, Y.; An, J.; Zhang, J. J. Environ. Sci.-China 2010, 31, 10 (in Chinese). (吴方斿, 王跃思, 安俊琳, 张俊刚, 环境科学, 2010, 31, 10.)

[9] Zhang, J.; Zhao, Y.; Zhao, Q.; Shen, G.; Liu, Q.; Li, C.; Zhou, D.; Wang, S. Atmosphere 2018, 9, 373.

[10] Yu, D.; Tan, Z.; Lu, K.; Ma, X.; Li, X.; Chen, S.; Zhu, B.; Lin, L.; Li, Y.; Qiu, P.; Yang, X.; Liu, Y.; Wang, H.; He, L.; Huang, X.; Zhang, Y. Atmos. Environ. 2020, 224, 117304.

[11] Song, M.; Tan, Q.; Feng, M.; Qu, Y.; Liu, X.; An, J.; Zhang, Y. J. Geophys. Res-Atmos. 2018, 123, 9741.

[12] Wu, R.; Xie, S. Environ. Sci. Technol. 2018, 52, 8146.

[13] Ng, N. L.; Kroll, J. H.; Chan, A. W. H.; Chhabra, P. S.; Flagan, R. C.; Seinfeld, J. H. Atmos. Chem. Phys. 2007, 7, 3909.

[14] Kroflič, A.; Grilc, M.; Grgić, I. Sci. Rep. 2014, 5, 8859.

[15] Pflieger, M.; Kroflič, A. J. Hazard. Mater. 2017, 338, 132.

[16] Shiohara, N.; Fernández-Bremauntz, A. A.; Jiménez, S. B.; Yanagisawa, Y. Atmos. Environ. 2005, 39, 3481.

[17] Kuykendall, J. R.; Shaw, S. L.; Dennis, P.; Kurt, F.; Sam, K.; Victor, K. Inhal. Toxicol. 2009, 21, 747.

[18] Samet, J.; Chiu, W.; Cogliano, V.; Jinot, J.; Kriebel, D.; Lunn, R.; Beland, F.; Bero, L.; Browne, P.; Fritschi, L.; Kanno, J.; Lachenmeier, D.; Lan, Q.; Lasfargues, G.; Curieux, F.; Peters, S.; Shubat, P.; Sone, H.; White, M.; Wild, C. J. Natl. Cancer. I 2020, 112,30 .

[19] Duarte-Davidson, R.; Courage, C.; Rushton, L.; Levy, L. Occup. Environ. Med. 2001, 58, 2.

[20] Moolla, R.; Curtis, C. J.; Knight, J. 2015, 12, 4101.

[21] Lin, P.; Liu, J.; Shilling, J. E.; Kathmann, S. M.; Laskin, J.; Laskin, A. Phys. Chem. Chem. Phys. 2015, 17, 23312.

[22] Li, X.; Wang, Y.; Hu, M.; Tan, T.; Li, M.; Wu, Z.; Chen, S.; Tang, X. Atmos. Environ. 2020, 237, 117712

[23] Wang, Y.; Hu, M.; Li, X.; Xu, N. Prog. Chem. 2020, 32, 627 (in Chinese). (王玉珏, 胡敏, 李晓, 徐楠, 化学进展, 2020, 32, 627.)

[24] Wang, L.; Wu, R.; Xu, C. J. Phys. Chem. A 2013, 117, 14163.

[25] Arey, J.; Obermeyer, G.; Aschmann, S. M.; Chattopadhyay, S.; Cusick, R. D.; Atkinson, R. Environ. Sci. Technol. 2009, 43, 683.

[26] Zaytsev, A.; Koss, A. R.; Breitenlechner, M.; Krechmer, J. E.; Nihill, K. J.; Lim, C. Y.; Rowe, J. C.; Cox, J. L.; Moss, J.; Roscioli, J. R.; Canagaratna, M. R.; Worsnop, D. R.; Kroll, J. H.; Keutsch, F. N. Atmos. Chem. Phys. 2019, 19, 15117.

[27] Xu, L.; Moller, K. H.; Crounse, J. D.; Kjaergaard, H. G.; Wennberg, P. O. Environ. Sci. Technol. 2020, 54, 13467.

[28] Wang, S. N.; Newland, M. J.; Deng, W.; Rickard, A. R.; Hamilton, J. F.; Munoz, A.; Rodenas, M.; Vazquez, M. M.; Wang, L. M.; Wang, X. M. Environ. Sci. Technol. 2020, 54, 7798.

[29] Schwantes, R. H.; Schilling, K. A.; McVay, R. C.; Lignell, H.; Coggon, M. M.; Zhang, X.; Wennberg, P. O.; Seinfeld, J. H. Atmos. Chem. Phys. 2017, 17, 3453.

[30] Atkinson, R.; Aschmann, S. M.; Arey, J.; Carter, W. P. L. Int. J. Chem. Kinet. 1989, 21, 801.

[31] Baltaretu, C. O.; Lichtman, E. I.; Hadler, A. B.; Elrod, M. J. J. Phys. Chem. A 2009, 113, 221.

[32] Bandow, H.; Washida, N. Bull. Chem. Soc. Jpn. 1985, 58, 2541.

[33] Becker, K. H.; Barnes, I.; Bierbach, A.; Brockmann, K.; Kirchner, F.; Klotz, B.; Libuda, H.; Mayer-Figge, A.; Mönninghoff, S.; Ruppert, L. E. A.; Thomas, W.; Wiesen, E.; Wirtz, K.; Zabel, F. Chemical Processes in Atmospheric Oxidation, Eds.: Georges, L. B.,
Springer, Berlin, Heidelberg, 1997, p. 79.

[34] Birdsall, A. W.; Elrod, M. J. J. Phys. Chem. A 2011, 115, 5397.

[35] Dumdei, B. E.; Kenny, D. V.; Shepson, P. B.; Kleindienst, T. E.; Nero, C. M.; Cupitt, L. T.; Claxton, L. D. Environ. Sci. Technol. 1988, 22, 1493.

[36] Gery, M. W.; Fox, D. L.; Jeffries, H. E.; Stockburger, L.; Weathers, W. S. Int. J. Chem. Kinet. 1985, 17, 931.

[37] Gomez Alvarez, E.; Viidanoja, J.; Munoz, A.; Wirtz, K.; Hjorth, J. Environ. Sci. Technol. 2007, 41, 8362.

[38] Ji, Y.; Zhao, J.; Terazono, H.; Misawa, K.; Levitt, N. P.; Li, Y.; Lin, Y.; Peng, J.; Wang, Y.; Duan, L. Proc. Natl. Acad. Sci. U. S. A. 2017 $114,8169$.

[39] Klotz, B.; Sorensen, S.; Barnes, I.; Becker, K. H.; Etzkorn, T.; Volkamer, R.; Platt, U.; Wirtz, K.; Martin-Reviejo, M. J. Phys. Chem A 1998, 102, 10289.

[40] Moschonas, N.; Danalatos, D.; Glavas, S. Atmos. Environ. 1999, 33, 111.

[41] Nishino, N.; Arey, J.; Atkinson, R. J. Phys. Chem. A 2010, 114, 10140.

[42] Seuwen, R.; Warneck, P. Int. J. Chem. Kinet. 1996, $28,315$.

[43] Smith, D. F.; McIver, C. D.; Kleindienst, T. E. J. Atmos. Chem. 1998, $30,209$.

[44] Tuazon, E. C.; Macleod, H.; Atkinson, R.; Carter, W. P. L. Environ. Sci. Technol. 1986, 20, 383.

[45] Volkamer, R.; Platt, U.; Wirtz, K. J. Phys. Chem. A 2001, 105, 7865.

[46] Atkinson, R.; Carter, W. P. L.; Darnall, K. R.; Winer, A. M.; Pitts, J. N. Int. J. Chem. Kinet. 1980, 12, 779.

[47] Atkinson, R.; Carter, W. P. L.; Winer, A. M. J. Phys. Chem. 1983, 87, 1605.

[48] Shepson, P. B.; Edney, E. O.; Corse, E. W. J. Phys. Chem. 1984, 88, 4122.

[49] Tuazon, E. C.; Atkinson, R.; Macleod, H.; Biermann, H. W.; Winer, A. M.; Carter, W. P. L.; Pitts, J. N. Environ. Sci. Technol. 1984, 18, 981.

[50] Atkinson, R.; Aschmann, S. M. Int. J. Chem. Kinet. 1994, 26, 929.

[51] Wang, S.; Wu, R.; Berndt, T.; Ehn, M.; Wang, L. Environ. Sci. Technol. 2017, 51, 8442.

[52] Volkamer, R.; Jimenez, J. L.; San Martini, F.; Dzepina, K.; Zhang, Q.; Salcedo, D.; Molina, L. T.; Worsnop, D. R.; Molina, M. J. Geophys. Res. Lett. 2006, 33, L17811.

[53] Wang, Z.; Hu, M.; Wu, Z.; Yue, D. Acta Chim. Sinica 2013, 71, 519 (in Chinese). (王志涁, 胡敏, 吴志军, 岳玎利, 化学学报, 2013, 71, 519.)

[54] Guo, S.; Hu, M.; Shang, D.; Guo, Q.; Hu, W. Acta Chim. Sinica 2014, 72, 145 (in Chinese). (郭松, 胡敏, 尚冬杰, 郭庆丰, 胡伟伟, 化学学报, 2014, 72, 145.)

[55] Wang, H.; Yu, Y.; Tang, R.; Guo, S. Acta Chim. Sinica 2020, 78, 516 (in Chinese). (王辉, 俞渘, 唐荣志, 郭松, 化学学报, 2020, 78, 516.)

[56] Hoshino, M.; Akimoto, H.; Okuda, M. Bull. Chem. Soc. Jpn. 1978 51,718 .

[57] Uc, V. H.; García-Cruz, I.; Hernández-Laguna, A.; Vivier-Bunge, A. J. Phys. Chem. A 2000, 104, 7849.

[58] Bloss, C.; Wagner, V.; Bonzanini, A.; Jenkin, M. E.; Wirtz, K.; Martin-Reviejo, M.; Pilling, M. J. Atmos. Chem. Phys. 2005, 5, 623.

[59] Andino, J. M.; Vivier-Bunge, A. Adv. Quantum Chem. 2008, 55, 297.

[60] Birdsall, A. W.; Andreoni, J. F.; Elrod, M. J. J. Phys. Chem. A 2010, 114, 10655.

[61] Nehr, S.; Bohn, B.; Wahner, A. J. Phys. Chem. A 2011, 116, 6015.

[62] Wu, R.; Pan, S.; Li, Y.; Wang, L. J. Phys. Chem. A 2014, 118, 4533.

[63] Vereecken, L. Advances in Atmospheric Chemistry, World Scientific Publishing Co Pte Ltd., Singapore, 2019, pp. $377 \sim 527$.

[64] Lin, W.; Janet, A.; Roger, A. Environ. Sci. Technol. 2005, 39, 5302.

[65] Roger, A.; Janet, A. Chem. Rev. 2003, 103, 4605.

[66] Uc, V. H.; Alvarez-Idaboy, J. R.; Galano, A.; Vivier-Bunge, A. J. Phys. Chem. A 2008, 112, 7608.

[67] Molina, M. J.; Zhang, R.; Broekhuizen, K.; Lei, W.; Navarro, R.; Molina, L. T. J. Am. Chem. Soc. 1999, 121, 10225.

[68] Bohn, B. J. Phys. Chem. A 2001, 105, 6092.

[69] Suh, I.; Dan, Z.; Zhang, R.; Molina, L. T.; Molina, M. J. Chem. Phys. Lett. 2002, 364, 454.

[70] Fan, J.; Zhang, R. J. Phys. Chem. A 2008, 112, 4314.

[71] Huang, M.; Wang, Z.; Hao, L.; Zhang, W. Comput. Theor. Chem. 2011, 965, 285.

[72] Newland, M. J.; Jenkin, M. E.; Rickard, A. R. Proc. Natl. Acad. Sci. 
U. S. A. 2017, 114, E7856.

[73] Suh, I.; Zhang, R.; Molina, L. T.; Molina, M. J. J. Am. Chem. Soc. 2003, 125, 12655.

[74] Pan, S.; Wang, L. Acta Phys.-Chim. Sin. 2015, 31, 2259.

[75] Bartolotti, L. J.; Edney, E. O. Chem. Phys. Lett. 1995, 245, 119.

[76] Yu, J.; Jeffries, H. E.; Sexton, K. G. Atmos. Environ. 1997, 31, 2261.

[77] Ghigo, G.; Tonachini, G. J. Am. Chem. Soc. 1998, 120, 6753.

[78] Frankcombe, T. J.; Smith, S. C. J. Phys. Chem. A 2007, 111, 3686.

[79] Frankcombe, T. J.; Smith, S. C. J. Phys. Chem. A 2007, 111, 3691.

[80] Lay, T. H.; Bozzelli, J. W.; Seinfeld, J. H. J. Phys. Chem. 1996, 100, 6543.

[81] Koch, R.; Knispel, R.; Elend, M.; Siese, M.; Zetzsch, C. Atmos. Chem. Phys. 2007, 7, 2057.

[82] Pan, S.; Wang, L. J. Phys. Chem. A 2014, 118, 10778.

[83] Motta, F.; Ghigo, G.; Tonachini, G. J. Phys. Chem. A 2002, 106, 4411.

[84] Qian, X.; Shen, H.; Chen, Z. Atmos. Environ. 2019, 214, 116845.

[85] Perring, A. E.; Pusede, S. E.; Cohen, R. C. Chem. Rev. 2013, 113, 5848.

[86] Walker, H. M.; Stone, D.; Ingham, T.; Vaughan, S.; Cain, M.; Jones, R. L.; Kennedy, O. J.; McLeod, M.; Ouyang, B.; Pyle, J.; Bauguitte, S.; Bandy, B.; Forster, G.; Evans, M. J.; Hamilton, J. F.; Hopkins, J. R.; Lee, J. D.; Lewis, A. C.; Lidster, R. T.; Punjabi, S.; Morgan, W. T.; Heard, D. E. Atmos. Chem. Phys. 2015, 15, 8179.

[87] Bianchi, F.; Kurten, T.; Riva, M.; Mohr, C.; Rissanen, M. P.; Roldin, P.; Berndt, T.; Crounse, J. D.; Wennberg, P. O.; Mentel, T. F.; Wildt, J.; Junninen, H.; Jokinen, T.; Kulmala, M.; Worsnop, D. R.; Thornton, J. A.; Donahue, N.; Kjaergaard, H. G.; Ehn, M. Chem. Rev. 2019, 119, 3472.

[88] Jenkin, M. E.; Valorso, R.; Aumont, B.; Rickard, A. R.; Wallington, T. J. Atmos. Chem. Phys. 2018, 18, 9329.

[89] Boyd, A. A.; Lesclaux, R. Int. J. Chem. Kinet. 1997, 29, 323.

[90] Boyd, A. A.; Noziere, B.; Lesclaux, R. J. Chem. Soc., Faraday Trans. 1996, 92,201

[91] Boyd, A. A.; Villenave, E.; Lesclaux, R. Int. J. Chem. Kinet. 1999, 31,37 .

[92] Boyd, A. A.; Villenave, E.; Lesclaux, R. Atmos. Environ. 2003, 37, 2751.

[93] Glover, B. G.; Miller, T. A. J. Phys. Chem. A 2005, 109, 11191.

[94] Hansen, J. C.; Li, Y. M.; Rosado-Reyes, C. M.; Francisco, J. S.; Szente, J. J.; Maricq, M. M. J. Phys. Chem. A 2003, 107, 5306.

[95] Jenkin, M. E.; Boyd, A. A.; Lesclaux, R. J. Atmos. Chem. 1998, 29, 267.

[96] Jenkin, M. E.; Hayman, G. D. J. Chem. Soc., Faraday Trans. 1995, 91, 1911

[97] Jenkin, M. E.; Murrells, T. P.; Shalliker, S. J.; Hayman, G. D. J. Chem. Soc., Faraday Trans. 1993, 89, 433.

[98] Le Crane, I. P.; Villenave, E. Int. J. Chem. Kinet. 2006, 38, 276.

[99] Le Crane, J. P.; Villenave, E.; Hurley, M. D.; Wallington, T. J.; Nishida, S.; Takahashi, K.; Matsumi, Y. J. Phys. Chem. A 2004, 108, 795 .

[100] Lightfoot, P. D.; Cox, R. A.; Crowley, J. N.; Destriau, M.; Hayman, G. D.; Jenkin, M. E.; Moortgat, G. K.; Zabel, F. Atmos. Environ. 1992, 26, 1805.

[101] Noziere, B.; Hanson, D. R. J. Phys. Chem. A 2017, 121, 8453.

[102] Rowley, D. M.; Lightfoot, P. D.; Lesclaux, R.; Wallington, T. J. J. Chem. Soc., Faraday Trans. 1991, 87, 3221.

[103] Rowley, D. M.; Lightfoot, P. D.; Lesclaux, R.; Wallington, T. J. J. Chem. Soc., Faraday Trans. 1992, 88, 1369.

[104] Tomas, A.; Lesclaux, R. Chem. Phys. Lett. 2000, 319, 521

[105] Villenave, E.; Lesclaux, R. J. Phys. Chem. 1996, 100, 14372.

[106] Villenave, E.; Lesclaux, R.; Seefeld, S.; Stockwell, W. R. J. Geophys. Res.-Atmos. 1998, 103, 25273.

[107] Assaf, E.; Song, B.; Tomas, A.; Schoemaecker, C.; Fittschen, C. J. Phys. Chem. A 2016, 120, 8923.

[108] Assaf, E.; Tanaka, S.; Kajii, Y.; Schoemaecker, C.; Fittschen, C. Chem. Phys. Lett. 2017, 684, 245.

[109] deGouw, J. A.; Howard, C. J. J. Phys. Chem. A 1997, 101, 8662.

[110] Eberhard, J.; Howard, C. J. Int. J. Chem. Kinet. 1996, 28, 731

[111] Eberhard, J.; Howard, C. J. J. Phys. Chem. A 1997, 101, 3360

[112] Elrod, M. J. J. Phys. Chem. A 2011, 115, 8125.

[113] Farago, E. P.; Schoemaecker, C.; Viskolcz, B.; Fittschen, C. Chem. Phys. Lett. 2015, 619, 196.

[114] Hsin, H. Y.; Elrod, M. J. J. Phys. Chem. A 2007, 111, 613.

[115] Miller, A. M.; Yeung, L. Y.; Kiep, A. C.; Elrod, M. J. Phys. Chem.
Chem. Phys. 2004, 6, 3402.

[116] Yan, C.; Kocevska, S.; Krasnoperov, L. N. J. Phys. Chem. A 2016, $120,6111$.

[117] Andino, J. M.; Smith, J. N.; Flagan, R. C.; Goddard, W. A.; Seinfeld, J. H. J. Phys. Chem. 1996, 100, 10967.

[118] Suh, I.; Zhao, J.; Zhang, R. Chem. Phys. Lett. 2006, 432, 313.

[119] Berndt, T.; Boge, O. Phys. Chem. Chem. Phys. 2006, 8, 1205.

[120] Paulot, F.; Crounse, J. D.; Kjaergaard, H. G.; Kuerten, A.; St Clair, J. M.; Seinfeld, J. H.; Wennberg, P. O. Science 2009, 325, 730.

[121] Peeters, J.; Nguyen, T. L.; Vereecken, L. Phys. Chem. Chem. Phys. 2009, 11,5935 .

[122] Dawson, M. L.; Xu, J.; Griffin, R. J.; Dabdub, D. Geosci. Model. Dev. 2016, 9, 2143.

[123] Xu, J.; Griffin, R. J.; Liu, Y.; Nakao, S.; Cocker, D. R., III. Atmos. Environ. 2015, 101, 217.

[124] Le Breton, M.; McGillen, M. R.; Muller, J. B. A.; Bacak, A.; Shallcross, D. E.; Xiao, P.; Huey, L. G.; Tanner, D.; Coe, H.; Percival, C. J. Atmos. Meas. Tech. 2012, 5, 3029.

[125] Paulot, F.; Wunch, D.; Crounse, J. D.; Toon, G. C.; Millet, D. B.; DeCarlo, P. F.; Vigouroux, C.; Deutscher, N. M.; Abad, G. G.; Notholt, J.; Warneke, T.; Hannigan, J. W.; Warneke, C.; de Gouw, J. A.; Dunlea, E. J.; De Maziere, M.; Griffith, D. W. T.; Bernath, P.; Jimenez, J. L.; Wennberg, P. O. Atmos. Chem. Phys. 2011, 11, 1989.

[126] Priestley, M.; Bannan, T. J.; Le Breton, M.; Worrall, S. D.; Kang, S.; Pullinen, I.; Schmitt, S.; Tillmann, R.; Kleist, E.; Zhao, D.; Wildt, J.; Garmash, O.; Mehra, A.; Bacak, A.; Shallcross, D. E.; KiendlerScharr, A.; Hallquist, A. M.; Ehn, M.; Coe, H.; Percival, C. J.; Hallquist, M.; Mentel, T. F.; McFiggans, G. Atmos. Chem. Phys. 2021, 21, 3473

[127] Garmash, O.; Rissanen, M. P.; Pullinen, I.; Schmitt, S.; Kausiala, O.; Tillmann, R.; Zhao, D.; Percival, C.; Bannan, T. J.; Priestley, M.; Hallquist, A. M.; Kleist, E.; Kiendler-Scharr, A.; Hallquist, M.; Berndt, T.; McFiggans, G.; Wildt, J.; Mentel, T.; Ehn, M. Atmos. Chem. Phys. 2020, 20, 515 .

[128] Stirnweis, L.; Marcolli, C.; Dommen, J.; Barmet, P.; Frege, C.; Platt, S. M.; Bruns, E. A.; Krapf, M.; Slowik, J. G.; Wolf, R.; Prevot, A. S H.; Baltensperger, U.; El-Haddad, I. Atmos. Chem. Phys. 2017, 17, 5035

[129] Surratt, J. D.; Chan, A. W. H.; Eddingsaas, N. C.; Chan, M.; Loza, C. L.; Kwan, A. J.; Hersey, S. P.; Flagan, R. C.; Wennberg, P. O.; Seinfeld, J. H. Proc. Natl. Acad. Sci. U. S. A. 2010, 107, 6640.

[130] Qi, X.; Zhu, S.; Zhu, C.; Hu, J.; Lou, S.; Xu, L.; Dong, J.; Cheng, P. Sci. Total Environ. 2020, 727.

[131] Glowacki, D. R.; Wang, L.; Pilling, M. J. J. Phys. Chem. A 2009 , $113,5385$.

[132] Huang, M. Q.; Zhang, W. J.; Wang, Z. Y.; Hao, L. Q.; Zhao, W. W.; Liu, X. Y.; Long, B.; Fang, L. J. Mol. Struc.-Theochem. 2008, 862, 28.

[133] Li, Y.; Wang, L. Phys. Chem. Chem. Phys. 2014, 16, 17908.

[134] Ponnusamy, S.; Sandhiya, L.; Senthilkumar, K. New J. Chem. 2017, $41,10259$.

[135] Klotz, B. r.; Volkamer, R.; Hurley, M. D.; Andersen, M. P. S.; Nielsen, O. J.; Barnes, I.; Imamura, T.; Wirtz, K.; Becker, K.-H.; Platt, U.; Wallington, T. J.; Washida, N. Phys. Chem. Chem. Phys. 2002, 4, 4399.

[136] Huang, M.; Wang, Z.; Hao, L.; Zhang, W. J. Mol. Struc.-Theochem. 2010, $944,21$.

[137] Nehr, S.; Bohn, B.; Dorn, H. P.; Fuchs, H.; Haeseler, R.; Hofzumahaus, A.; Li, X.; Rohrer, F.; Tillmann, R.; Wahner, A. Atmos. Chem. Phys. 2014, 14, 6941.

[138] Deng, W.; Liu, T.; Zhang, Y.; Situ, S.; Hu, Q.; He, Q.; Zhang, Z.; Lu, S.; Bi, X.; Wang, X.; Boreave, A.; George, C.; Ding, X.; Wang, X. Atmos. Environ. 2017, 150, 67.

[139] Cocker, D. R.; Flagan, R. C.; Seinfeld, J. H. Environ. Sci. Technol. 2001, 35, 2594.

[140] Carter, W. P. L.; Iii, D. R. C.; Fitz, D. R.; Malkina, I. L.; Bumiller, K.; Sauer, C. G.; Pisano, J. T.; Bufalino, C.; Song, C. Atmos. Environ. 2005, 39, 7768.

[141] Presto, A. A.; Hartz, K. E. H.; Donahue, N. M. Environ. Sci. Technol. 2005, 39, 7036.

[142] Platt, S. M.; El Haddad, I.; Zardini, A. A.; Clairotte, M.; Astorga, C.; Wolf, R.; Slowik, J. G.; Temime-Roussel, B.; Marchand, N.; Ježek, I.; Drinovec, L.; Močnik, G.; Möhler, O.; Richter, R.; Barmet, P.; Bianchi, F.; Baltensperger, U.; Prévôt, A. S. H. Atmos. Chem. Phys. 2013, 13, 9141 . 
[143] Hu, D.; Kamens, R. M. Atmos. Environ. 2007, 41, 6465.

[144] Kang, E.; Toohey, D. W.; Brune, W. H. Atmos. Chem. Phys. 2011, $11,1837$.

[145] Kang, E.; Root, M. J.; Toohey, D. W.; Brune, W. H. Atmos. Chem. Phys. 2007, 7, 5727.

[146] Yang, X.; Wang, H.; Tan, Z.; Lu, K.; Zhang, Y. Acta Chim. Sinica 2019, 77, 613 (in Chinese). (杨新平, 王海潮, 谭照峰, 陆克定, 张 远航, 化学学报, 2019, 77, 613.)

[147] Wang, T.; Španěl, P.; Smith, D. Int. J. Mass Spectrom. 2004, 239, 139.

[148] Hak, C.; Pundt, I.; Trick, S.; Kern, C.; Platt, U.; Dommen, J.; Ordonez, C.; Prevot, A. S. H.; Junkermann, W.; Astorga-Llorens, C.; Larsen, B. R.; Mellqvist, J.; Strandberg, A.; Yu, Y.; Galle, B.; Kleffmann, J.; Lorzer, J. C.; Braathen, G. O.; Volkamer, R. Atmos. Chem. Phys. 2005, 5, 2881.

[149] Fuchs, H.; Holland, F.; Hofzumahaus, A. Rev. Sci. Instrum. 2008, 79, 084104.

[150] Washenfelder, R. A.; Langford, A. O.; Fuchs, H.; Brown, S. S. Atmos. Chem. Phys. 2008, 8, 7779.

[151] Thalman, R.; Volkamer, R. Atmos. Meas. Tech. 2010, 3, 1797.

[152] Aufmhoff, H.; Hanke, M.; Uecker, J.; Schlager, H.; Arnold, F. Int. J. Mass Spectrom. 2011, 308, 26.

[153] Hornbrook, R. S.; Crawford, J. H.; Edwards, G. D.; Goyea, O.; Mauldin, R. L., III; Olson, J. S.; Cantrell, C. A. Atmos. Meas. Tech. 2011, 4, 735 .

[154] Fuchs, H.; Dorn, H. P.; Bachner, M.; Bohn, B.; Brauers, T.; Gomm, S.; Hofzumahaus, A.; Holland, F.; Nehr, S.; Rohrer, F.; Tillmann, R.; Wahner, A. Atmos. Meas. Tech. 2012, 5, 1611.

[155] Jokinen, T.; Sipila, M.; Junninen, H.; Ehn, M.; Lonn, G.; Hakala, J.; Petaja, T.; Mauldin, R. L., III; Kulmala, M.; Worsnop, D. R. Atmos. Chem. Phys. 2012, 12, 4117.

[156] Ehn, M.; Thornton, J. A.; Kleist, E.; Sipila, M.; Junninen, H.; Pullinen, I.; Springer, M.; Rubach, F.; Tillmann, R.; Lee, B.; Lopez-Hilfiker, F.; Andres, S.; Acir, I.-H.; Rissanen, M.; Jokinen, T.; Schobesberger, S.; Kangasluoma, J.; Kontkanen, J.; Nieminen, T.; Kurten, T.; Nielsen, L. B.; Jorgensen, S.; Kjaergaard, H. G.; Canagaratna, M.; Dal Maso, M.; Berndt, T.; Petaja, T.; Wahner, A. Kerminen, V.-M.; Kulmala, M.; Worsnop, D. R.; Wildt, J.; Mentel,
T. F. Nature 2014, 506, 476.

[157] Lee, B. H.; Lopez-Hilfiker, F. D.; Mohr, C.; Kurten, T.; Worsnop, D. R.; Thornton, J. A. Environ. Sci. Technol. 2014, 48, 6309.

[158] Pang, X.; Lewis, A. C.; Rickard, A. R.; Baeza-Romero, M. T.; Adams, T. J.; Ball, S. M.; Daniels, M. J. S.; Goodall, I. C. A.; Monks, P. S.; Peppe, S.; Rodenas Garcia, M.; Sanchez, P.; Munoz, A. Atmos. Meas. Tech. 2014, 7, 373.

[159] Veres, P. R.; Roberts, J. M.; Wild, R. J.; Edwards, P. M.; Brown, S. S.; Bates, T. S.; Quinn, P. K.; Johnson, J. E.; Zamora, R. J.; de Gouw, J. Atmos. Chem. Phys. 2015, 15, 8101.

[160] Lopez-Hilfiker, F. D.; Iyer, S.; Mohr, C.; Lee, B. H.; D'Ambro, E. L.; Kurten, T.; Thornton, J. A. Atmos. Meas. Tech. 2016, 9, 1505.

[161] Sanchez, J.; Tanner, D. J.; Chen, D.; Huey, L. G.; Ng, N. L. Atmos. Meas. Tech. 2016, 9, 3851.

[162] Thieser, J.; Schuster, G.; Schuladen, J.; Phillips, G. J.; Reiffs, A.; Parchatka, U.; Pöhler, D.; Lelieveld, J.; Crowley, J. N. Atmos. Meas. Tech. 2016, 9, 553 .

[163] Stoenner, C.; Derstroff, B.; Kluepfel, T.; Crowley, J. N.; Williams, J. J. Mass Spectrom. 2017, 52, 30.

[164] Hansel, A.; Scholz, W.; Mentler, B.; Fischer, L.; Berndt, T. Atmos. Environ. 2018, 186, 248.

[165] Albrecht, S. R.; Novelli, A.; Hofzumahaus, A.; Kang, S.; Baker, Y.; Mentel, T.; Wahner, A.; Fuchs, H. Atmos. Meas. Tech. 2019, 12, 891.

[166] Liu, J.; Li, X.; Yang, Y.; Wang, H.; Wu, Y.; Lu, X.; Chen, M.; Hu, J.; Fan, X.; Zeng, L.; Zhang, Y. Atmos. Meas. Tech. 2019, 12, 4439.

[167] Riva, M.; Rantala, P.; Krechmer, J. E.; Perakyla, O.; Zhang, Y.; Heikkinen, L.; Garmash, O.; Yan, C.; Kulmala, M.; Worsnop, D.; Ehn, M. Atmos. Meas. Tech. 2019, 12, 2403.

[168] Li, C.; Wang, H.; Chen, X.; Zhai, T.; Chen, S.; Li, X.; Zeng, L.; Lu, K. Atmos. Meas. Tech. 2021, 14, 4033.

[169] McFiggans, G.; Mentel, T. F.; Wildt, J.; Pullinen, I.; Kang, S.; Kleist, E.; Schmitt, S.; Springer, M.; Tillmann, R.; Wu, C.; Zhao, D.; Hallquist, M.; Faxon, C.; Le Breton, M.; Hallquist, A. M.; Simpson, D.; Bergstrom, R.; Jenkin, M. E.; Ehn, M.; Thornton, J. A.; Alfarra, M. R.; Bannan, T. J.; Percival, C. J.; Priestley, M.; Topping, D.; Kiendler-Scharr, A. Nature 2019, 565, 587.

(Cheng, B.) 